\title{
Electrophysiological Correlates of Listening to the Recitation of Quran
}

\section{Keivan Majidzadeh Ardabili' ${ }^{1}$, Reza Rostami², Reza Kazemi ${ }^{1 *}$}

${ }^{1}$ Tasnim Biotechnology Research Center (TBRC), Faculty of Medicine, AJA University of Medical Sciences, Tehran, Iran ${ }^{2}$ Department of Psychology, Faculty of Psychology and Education, University of Tehran, Tehran, Iran

\section{A BSTRACT}

Introduction: Spirituality and religion have an important role in human health and behavior. Referring to religious sources are one of the solutions that people seek during experience of life stress. Listening to the recitation of Quran has been suggested to alleviate the life $\mathrm{s}$ tress. The aim of this study was to determine the effects of listening to the recitation of the holy Quran on the brain functions in healthy people. Materials and Methods: 52 healthy subjects were recruited based on convenience sampling and were randomly placed into two groups. Quantitative Electroencephalography (qEEG) was recorded while the experimental group were listening to surah Al-Insan (experimental group), control group were listening to an Arabic text (control group). Brain activities were recorded and analyzed. Results: There were differences between the brain waves of the experimental and control groups. In the experimental group, all EEG frequency bands were decreased and the most of frequency bands changes observed in fronto-temporal pathway. In the control group, decrease in beta, high beta, gamma, gamma 1, gamma 2 and high gamma was evident in the central regions. In comparison to the control group, beta and gamma frequency bands increased in the experimental group. Conclusion: Changes in the EEG frequency bands of fronto-temporal pathway of listening to the recitation of Quran suggest the potential use of that in alleviation of psychological disorders.

\section{Key words:}

1. Neurosciences

2. Spirituality

3. Brain waves

*Corresponding Author: Reza Kazemi

E-mail: rezakazemi@ut.ac.ir 


\title{
همبستههاى الكتروفيزيولوزيك شنيدن تلاوت قر آن كريم
}

\author{
كيوان مجيدزاده اردبيلى'، رضا رستمى'، رضا كاظمى'" \\ 'مركز يزوهشى بيوتكنولوزى تسنيم، دانشكده يزشكى، دانشگاه علوم يزشكى ارتش، تهران، ايران

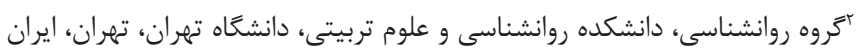

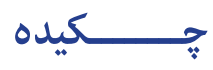

مقدمه: معنويت و مذهب نقش مهمى در سلامت و رفتار انسان دارند. مراجعه به منابع مذهبى يكى از

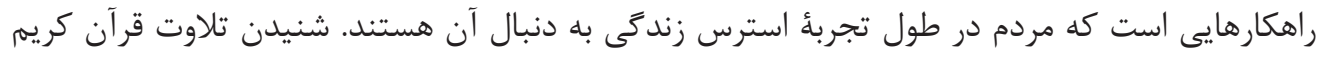

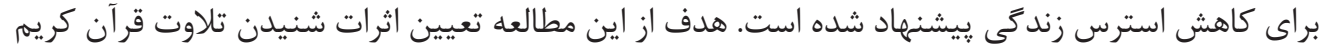

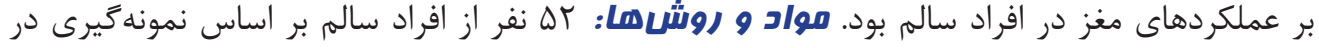

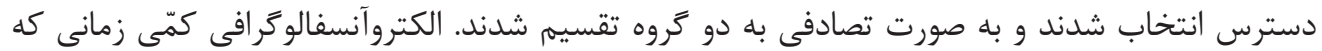

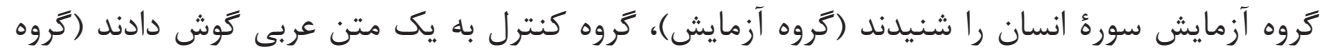

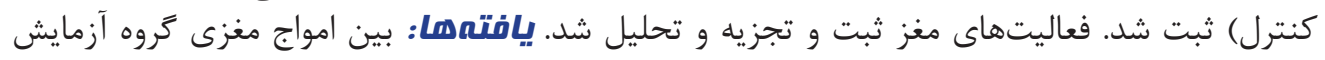

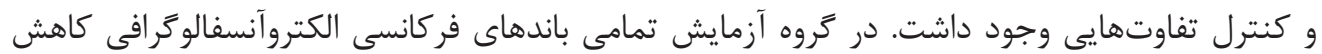

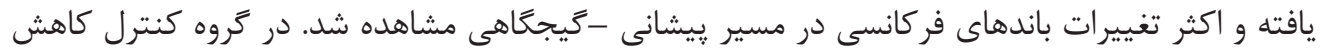

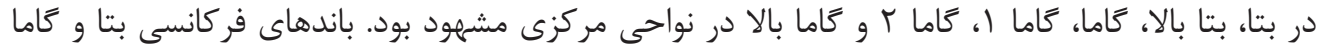

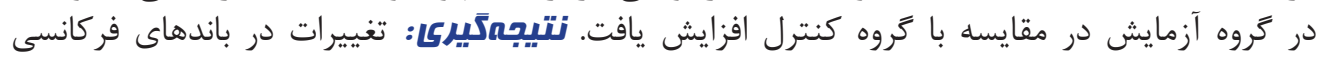

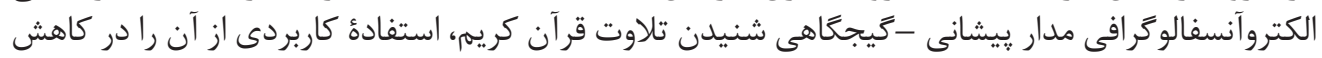

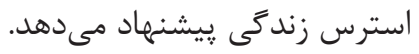

\footnotetext{
كليد وازهها:

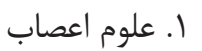

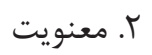

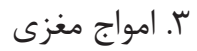

" نويسنده مسئول: رضا كاظمى , رئر

آدرس الكترونيكى: rezakazemi@ut.ac.ir 


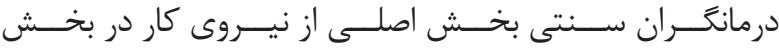
مقدمه

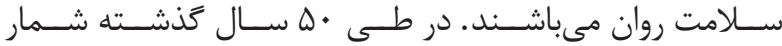

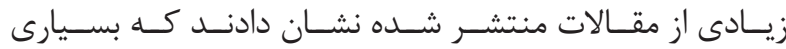

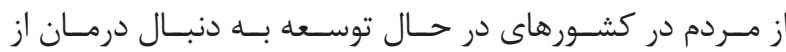

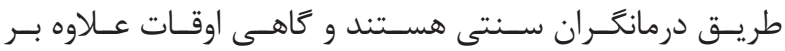

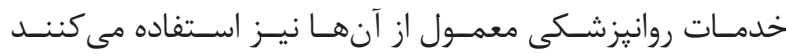

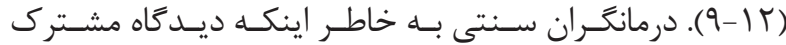

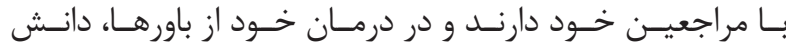

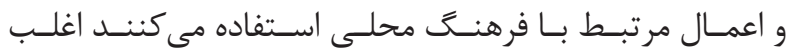

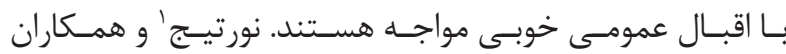

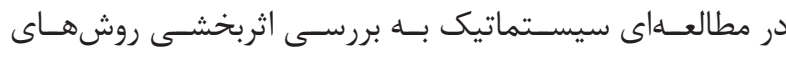

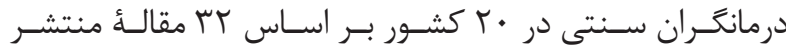

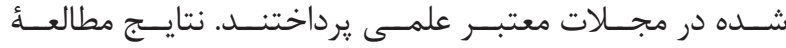

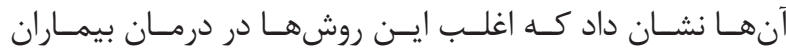

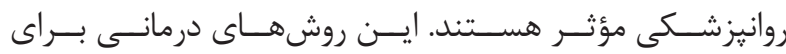

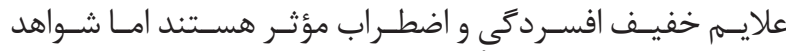

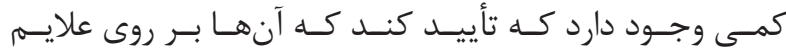

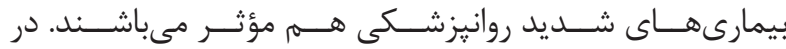

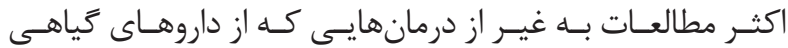

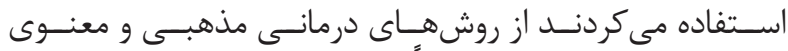

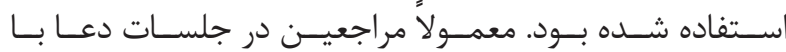

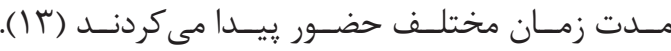

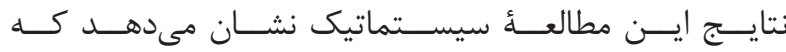

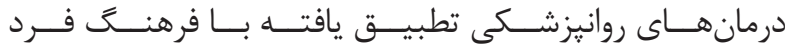

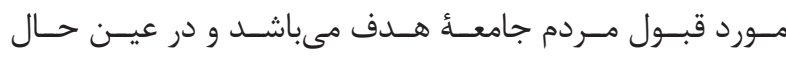

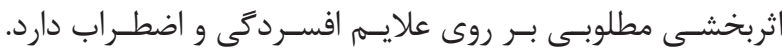

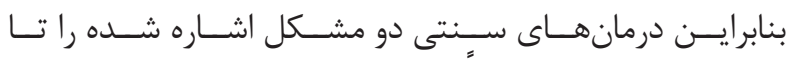

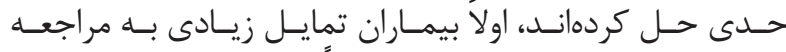

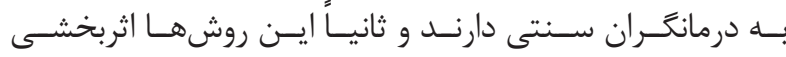

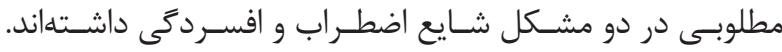

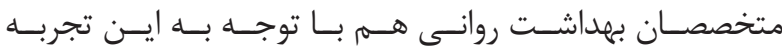

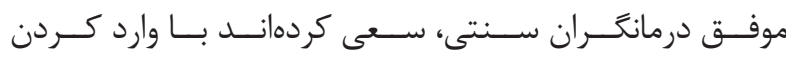

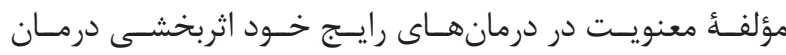

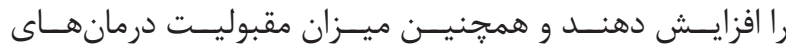

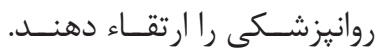

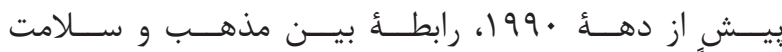

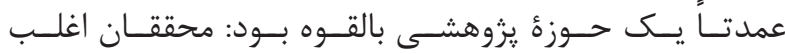

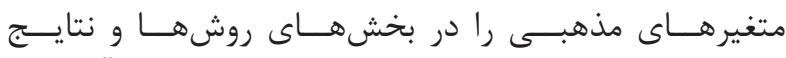

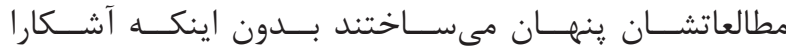

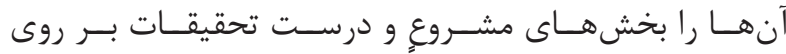

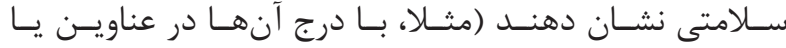

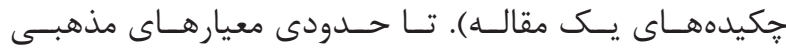

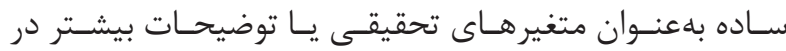

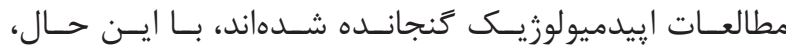

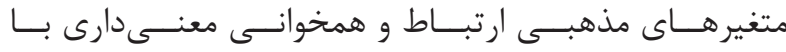

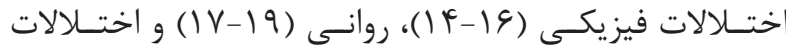

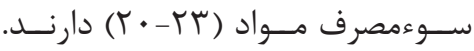

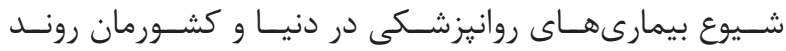

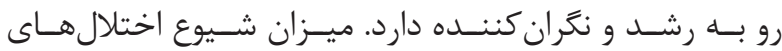

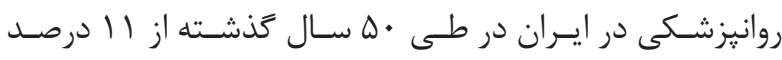

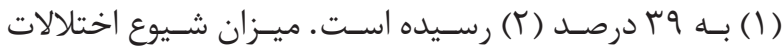

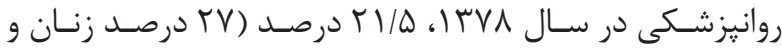

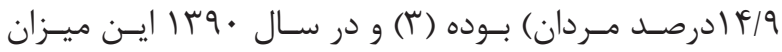

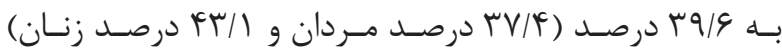

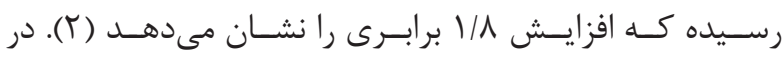

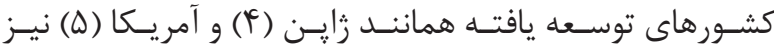

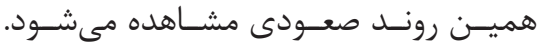

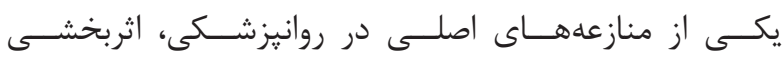

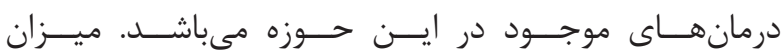

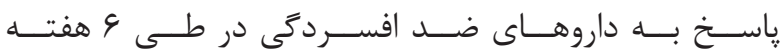

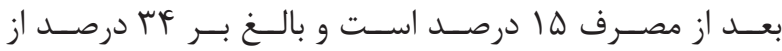

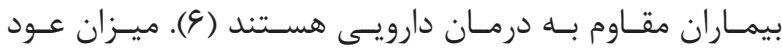

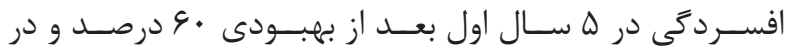

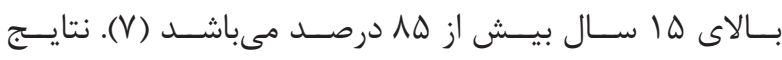

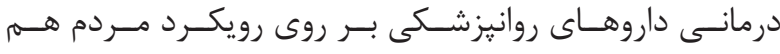

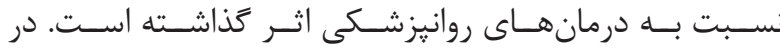

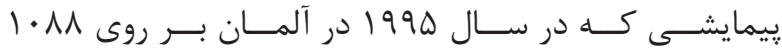

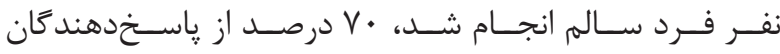

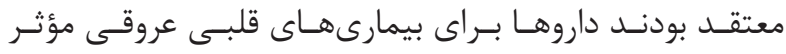

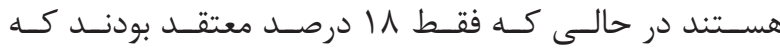

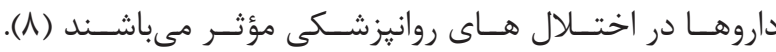

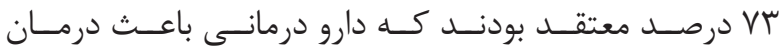

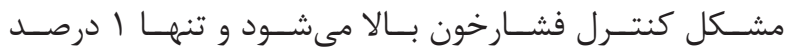

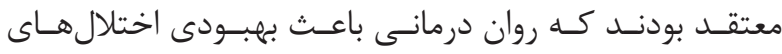

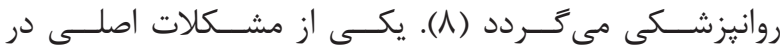

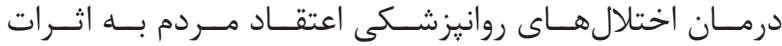

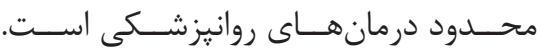

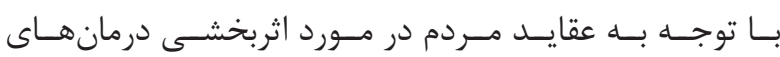

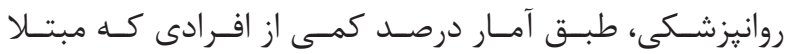

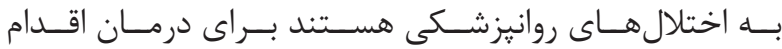

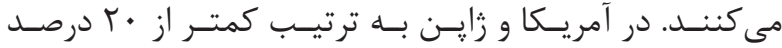

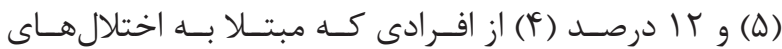

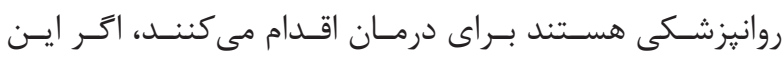

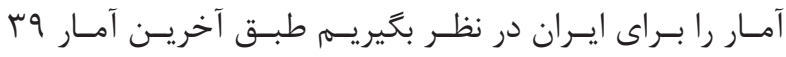

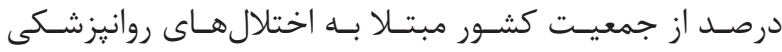

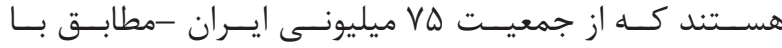

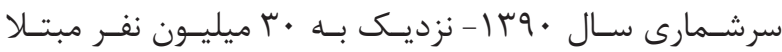

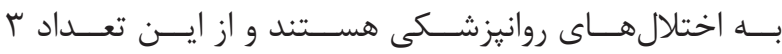

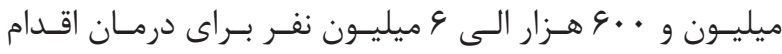

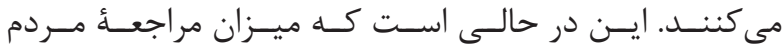

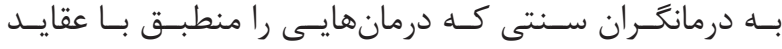

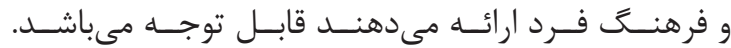


داد كـهـ ذهـن آكاهـى باعـث افزايسش آلفـاو وتتـا در مقايســهـ

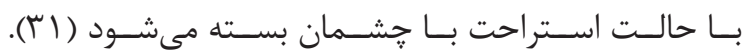

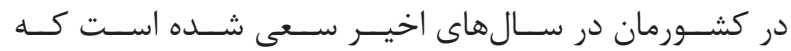

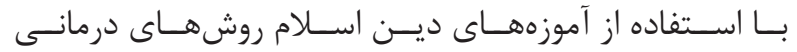

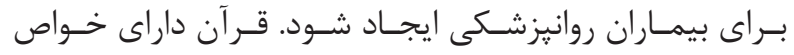

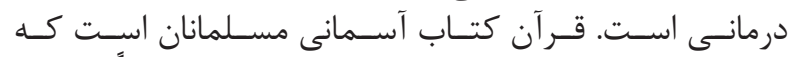

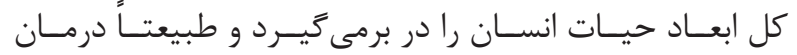

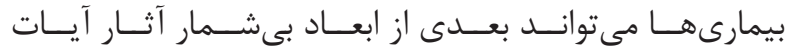

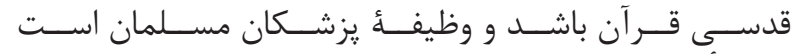

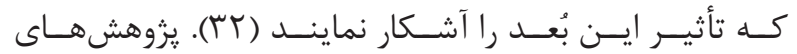

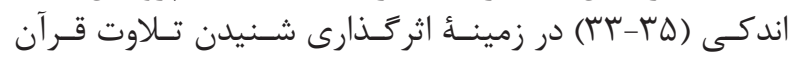

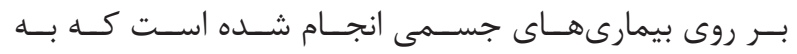

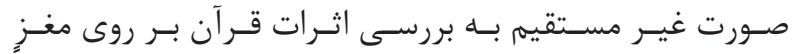

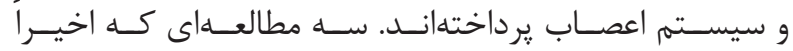

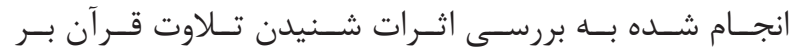

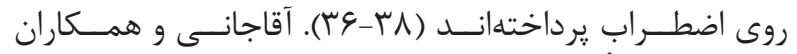

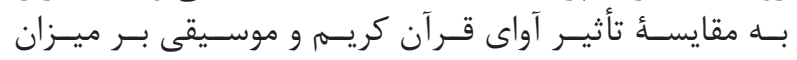

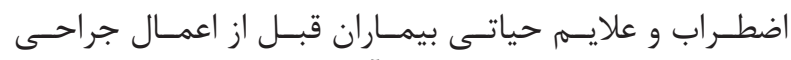

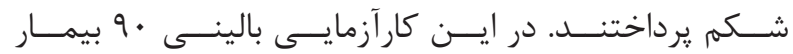

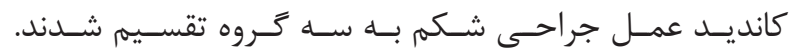

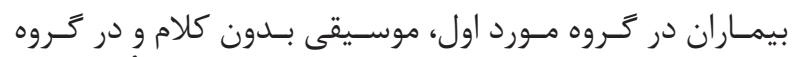

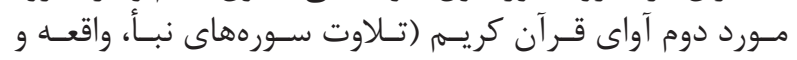

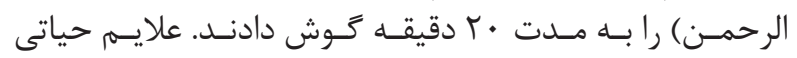

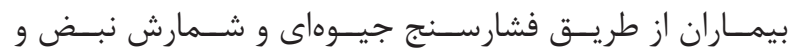

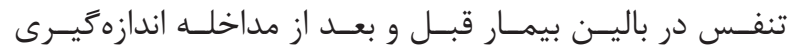

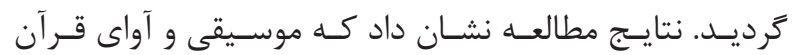

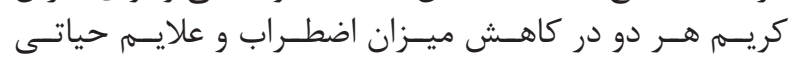

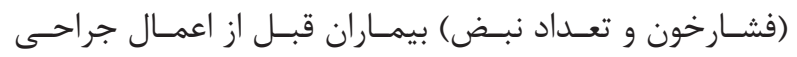

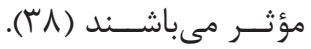

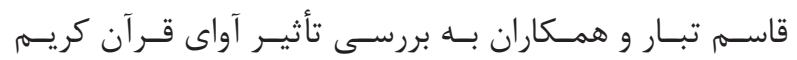

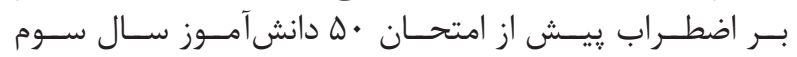

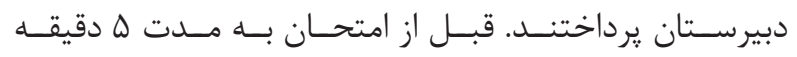

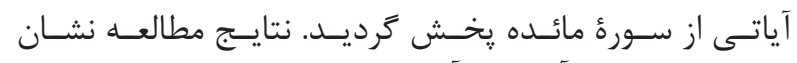

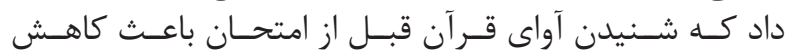

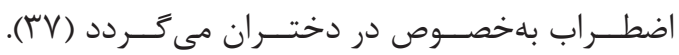

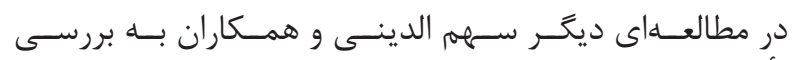

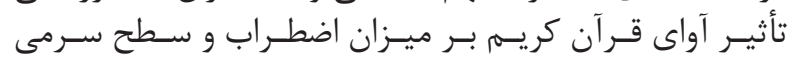

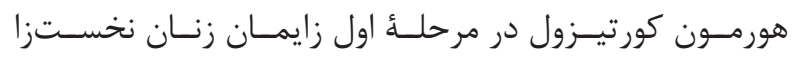

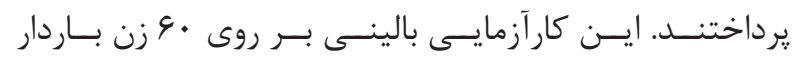

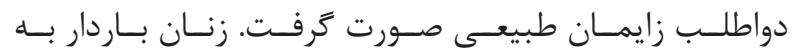

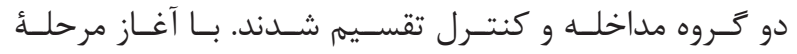

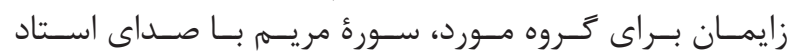

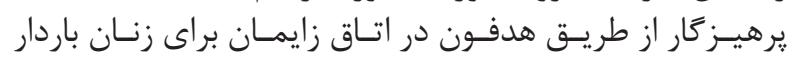

\footnotetext{
${ }^{2}$ Methodology

${ }^{3}$ Spiritual neuroscience

${ }^{4}$ Neurotheology

${ }^{5}$ Medial orbitofrontal cortex

${ }^{6}$ Middle temporal cortex

${ }^{7}$ Inferior and superior lobules

${ }^{8}$ Caudate

${ }^{9}$ Mindfullness
}

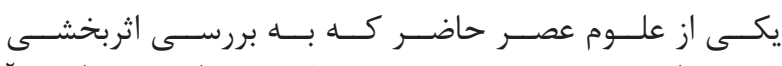

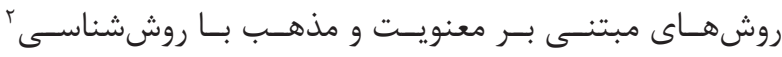

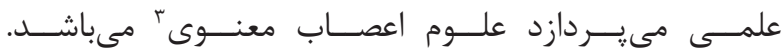

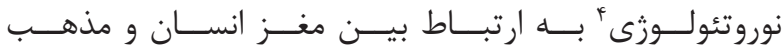

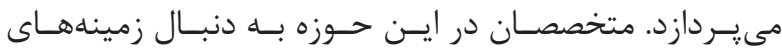

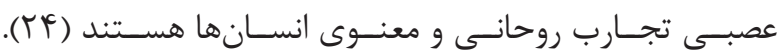

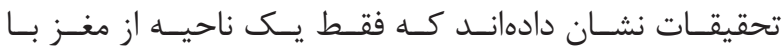

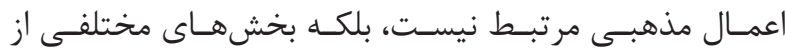

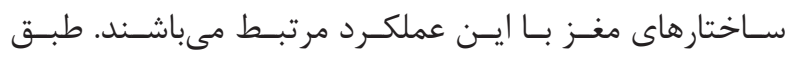

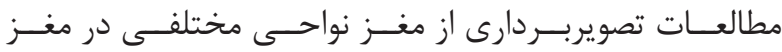

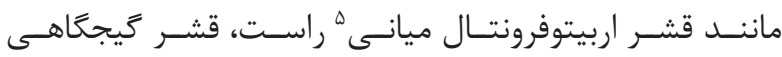

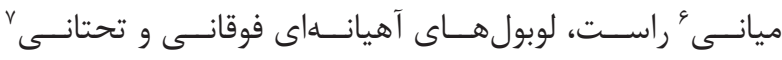

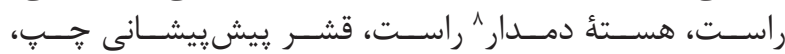

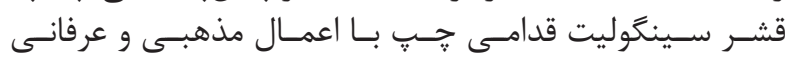

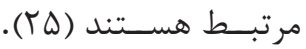

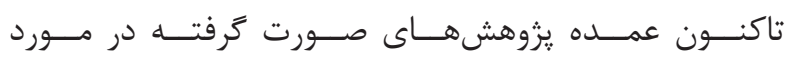

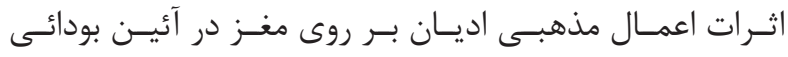

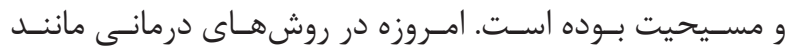

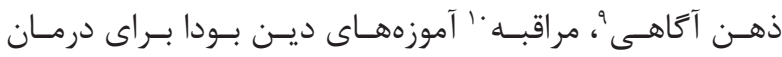

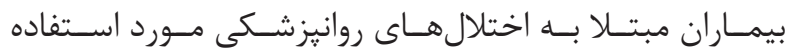

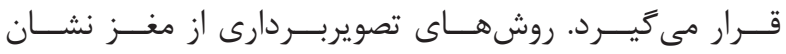

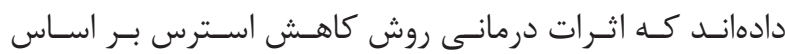

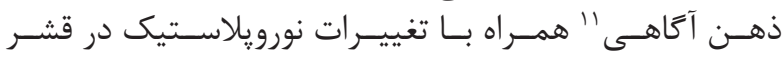

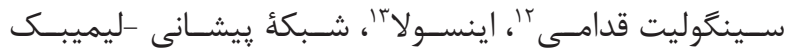

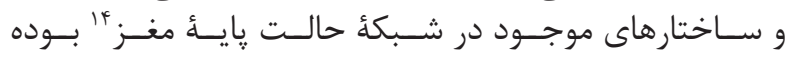

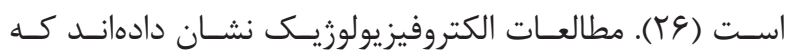

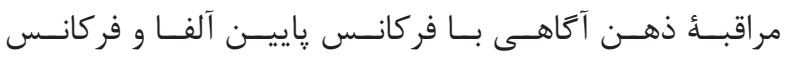

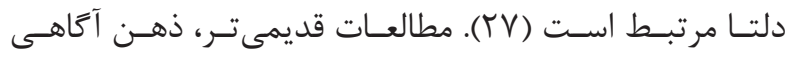

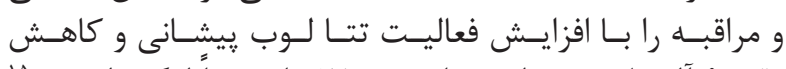

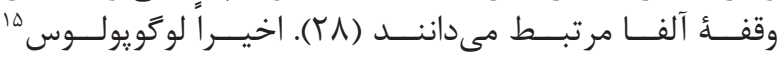

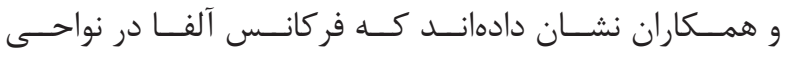

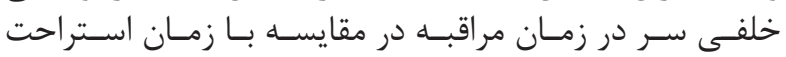

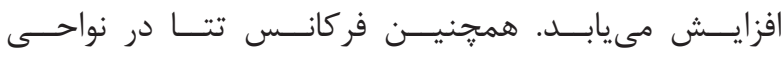

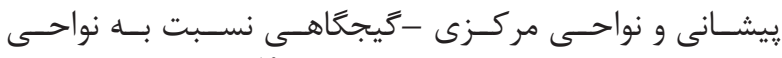

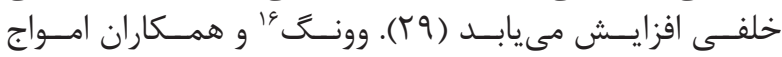

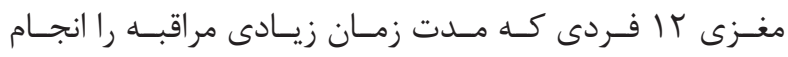

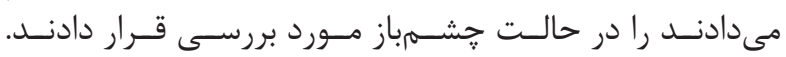

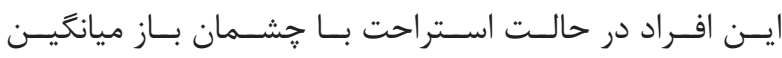

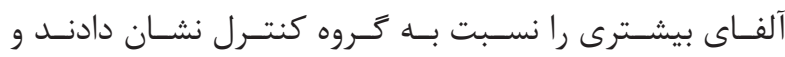

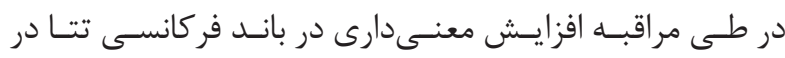

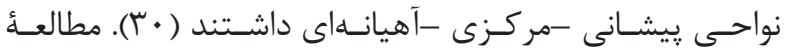

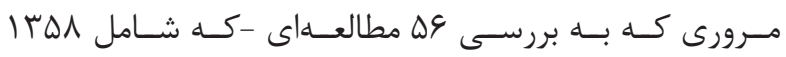

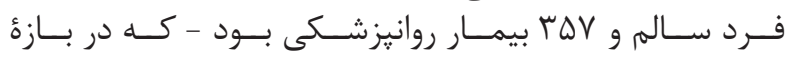

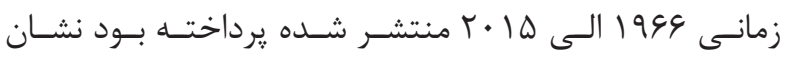

\footnotetext{
${ }^{10}$ Meditation

${ }^{11}$ Mindfullness based stress reduction

${ }^{12}$ Anterior cingulate

${ }^{13}$ Insula

${ }^{14}$ Default mode network

${ }^{15}$ Lagopoulos

${ }^{16}$ Wong
} 


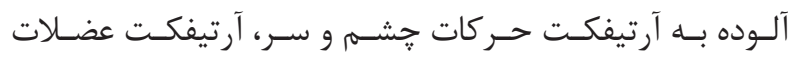

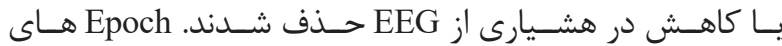

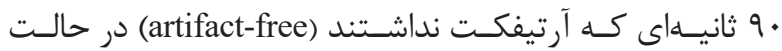

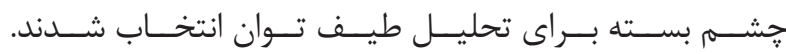

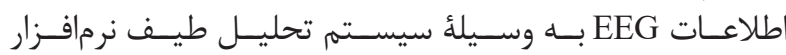

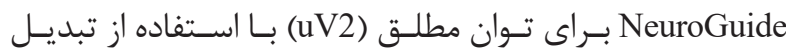

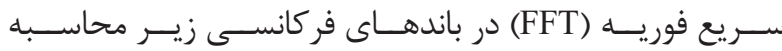

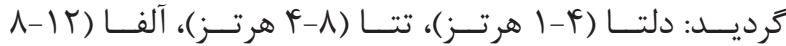

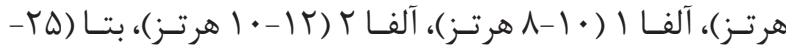

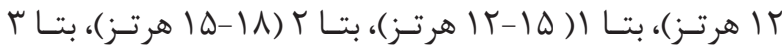

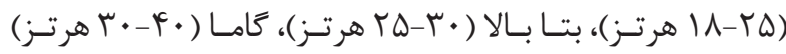

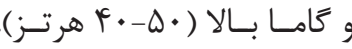

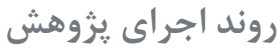

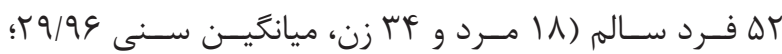

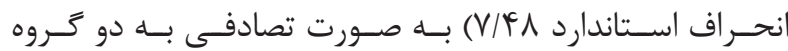

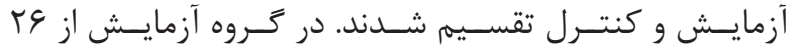

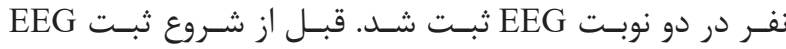

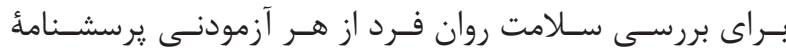
SCL90

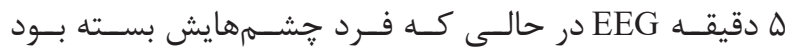

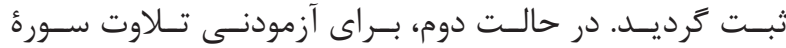

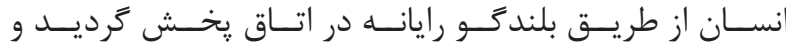

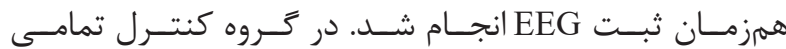

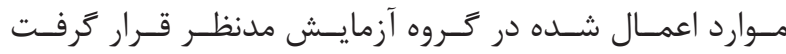

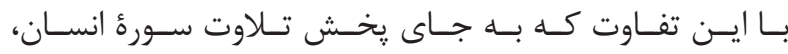

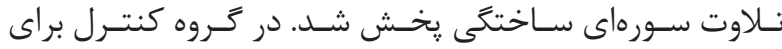

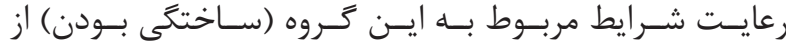

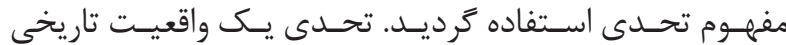

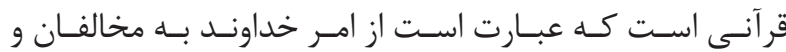

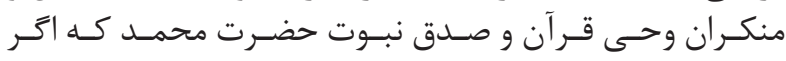

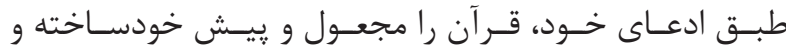

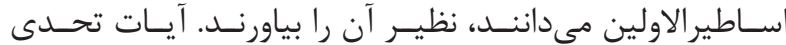

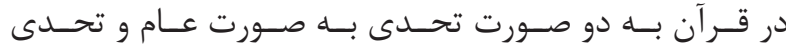

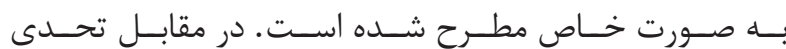

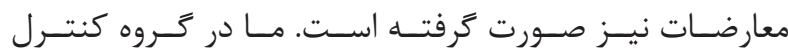

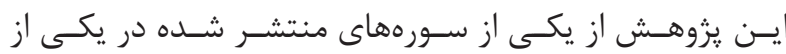

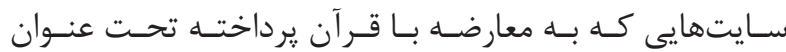

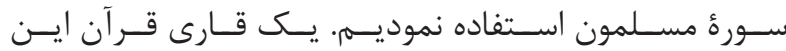

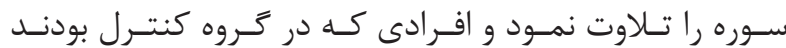

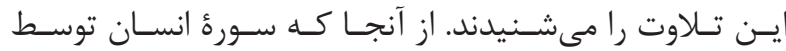

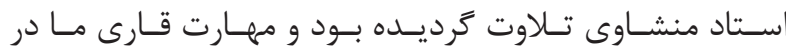

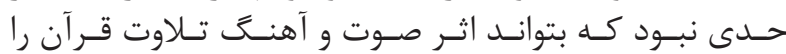

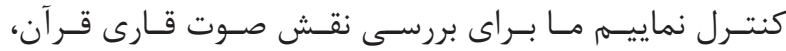

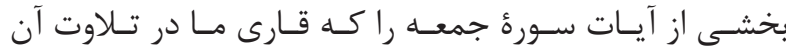

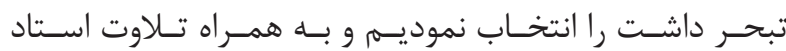

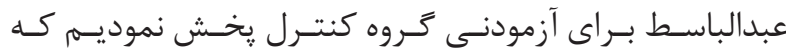

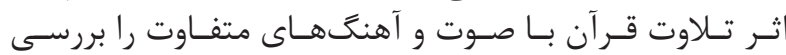

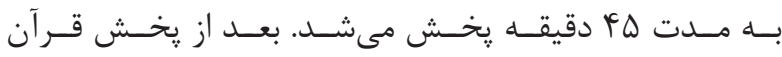

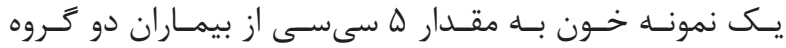

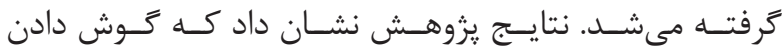

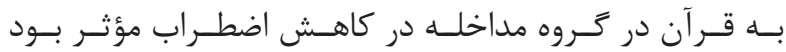

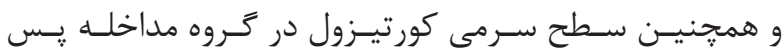

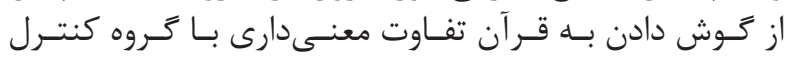

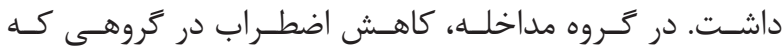

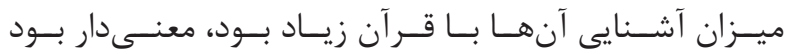

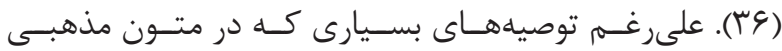

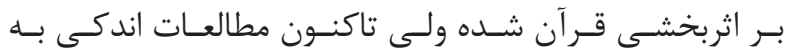

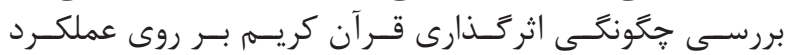

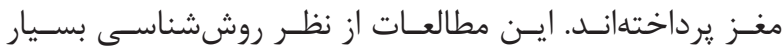

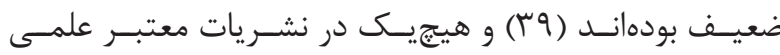

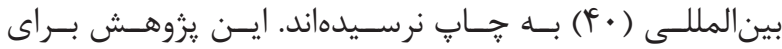

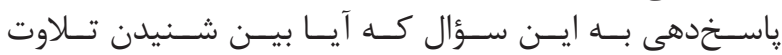

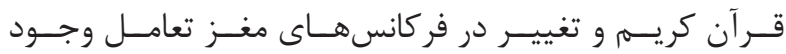

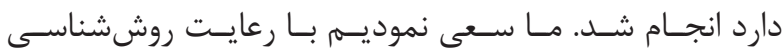

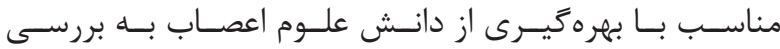

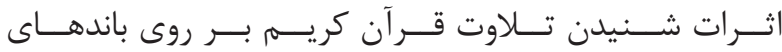
فر كانسى مغـز بيردازيسهم.

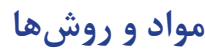

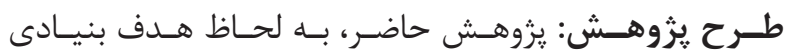

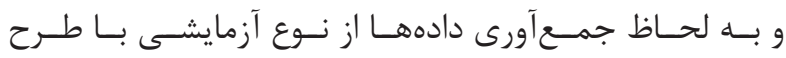

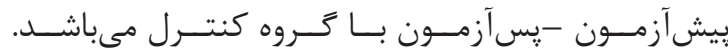

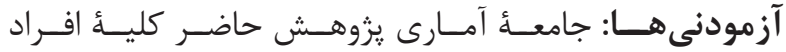

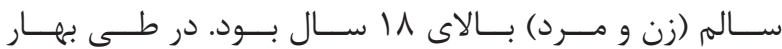

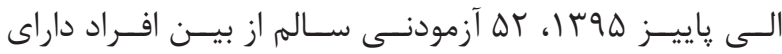

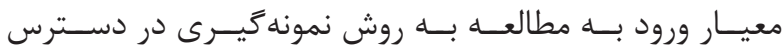

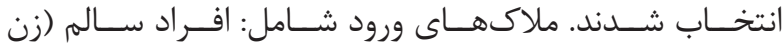

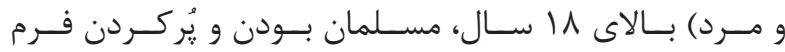

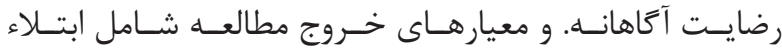

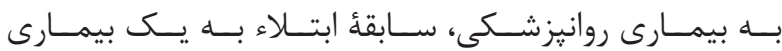

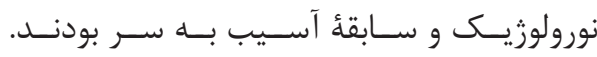

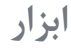

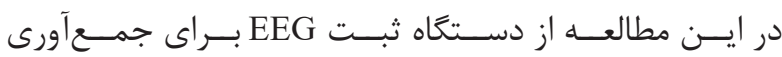
دادهـــا اسـتفاده كرديــد.

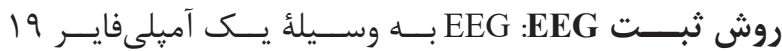

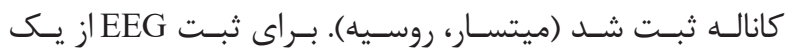

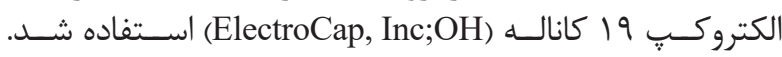

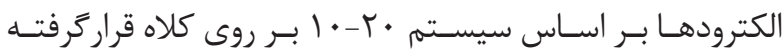

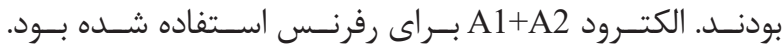

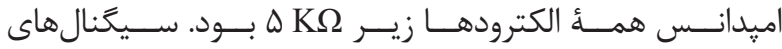

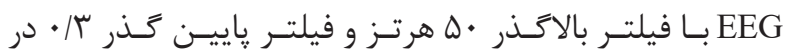

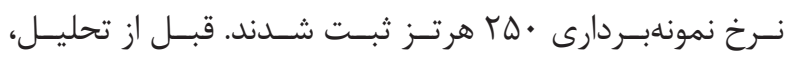

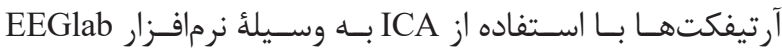

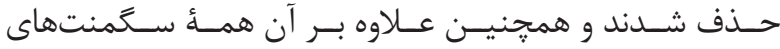




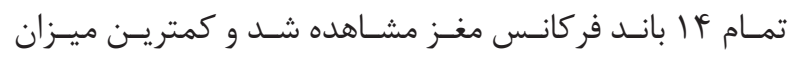

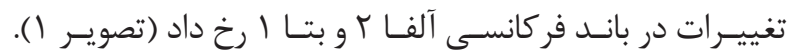

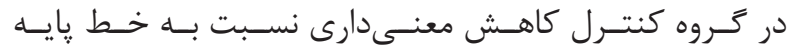

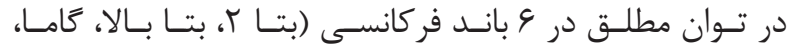

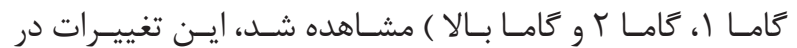

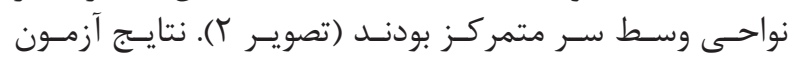

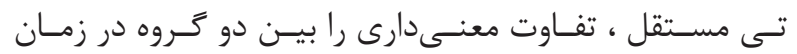

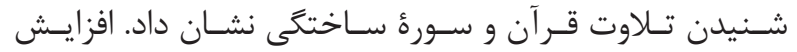

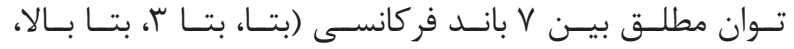

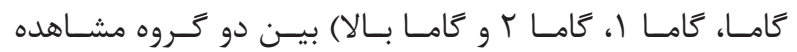

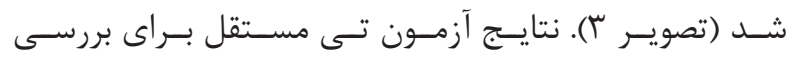

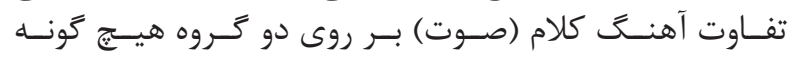

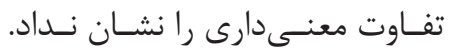

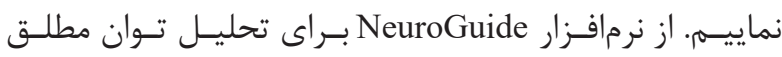

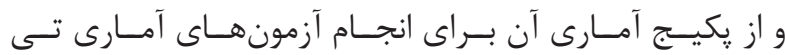

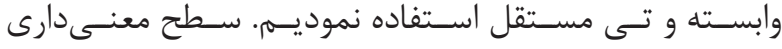

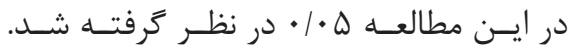
نافتهها

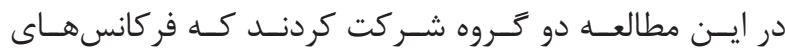

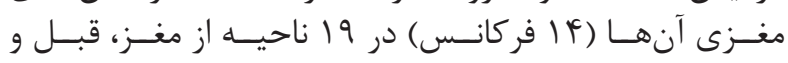

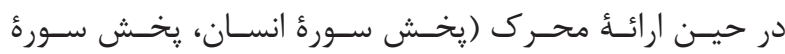

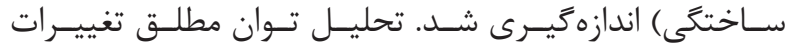

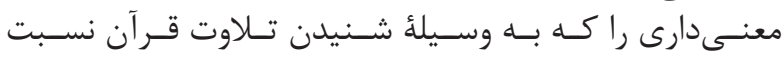

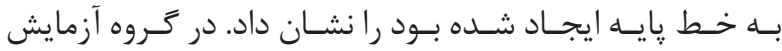

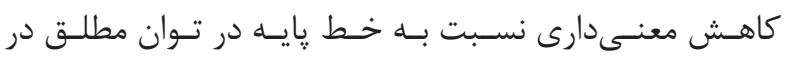

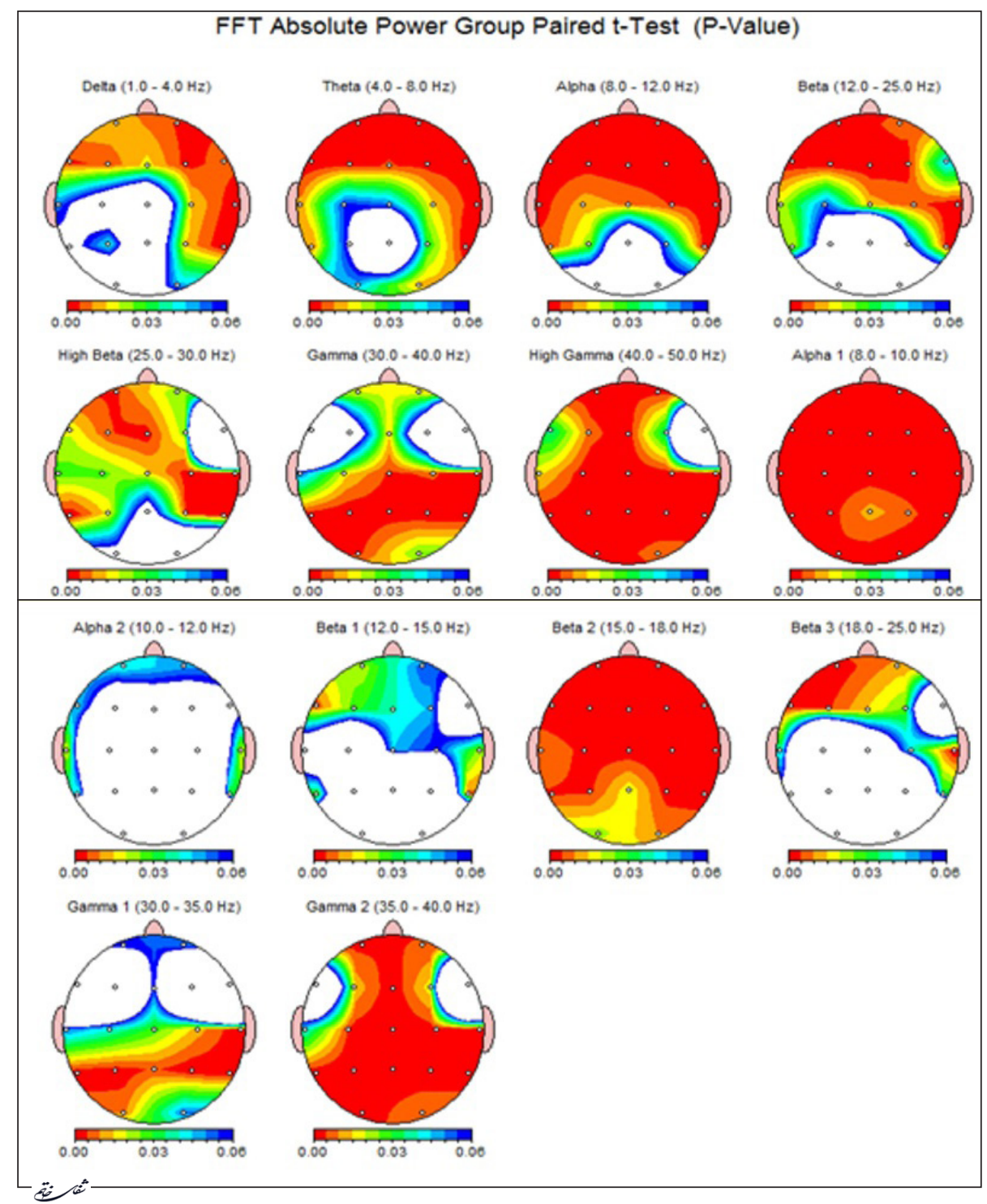

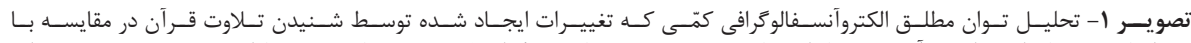

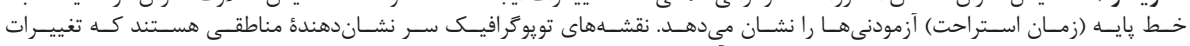

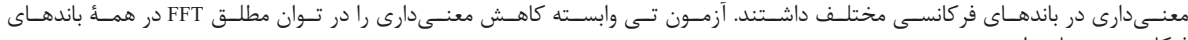




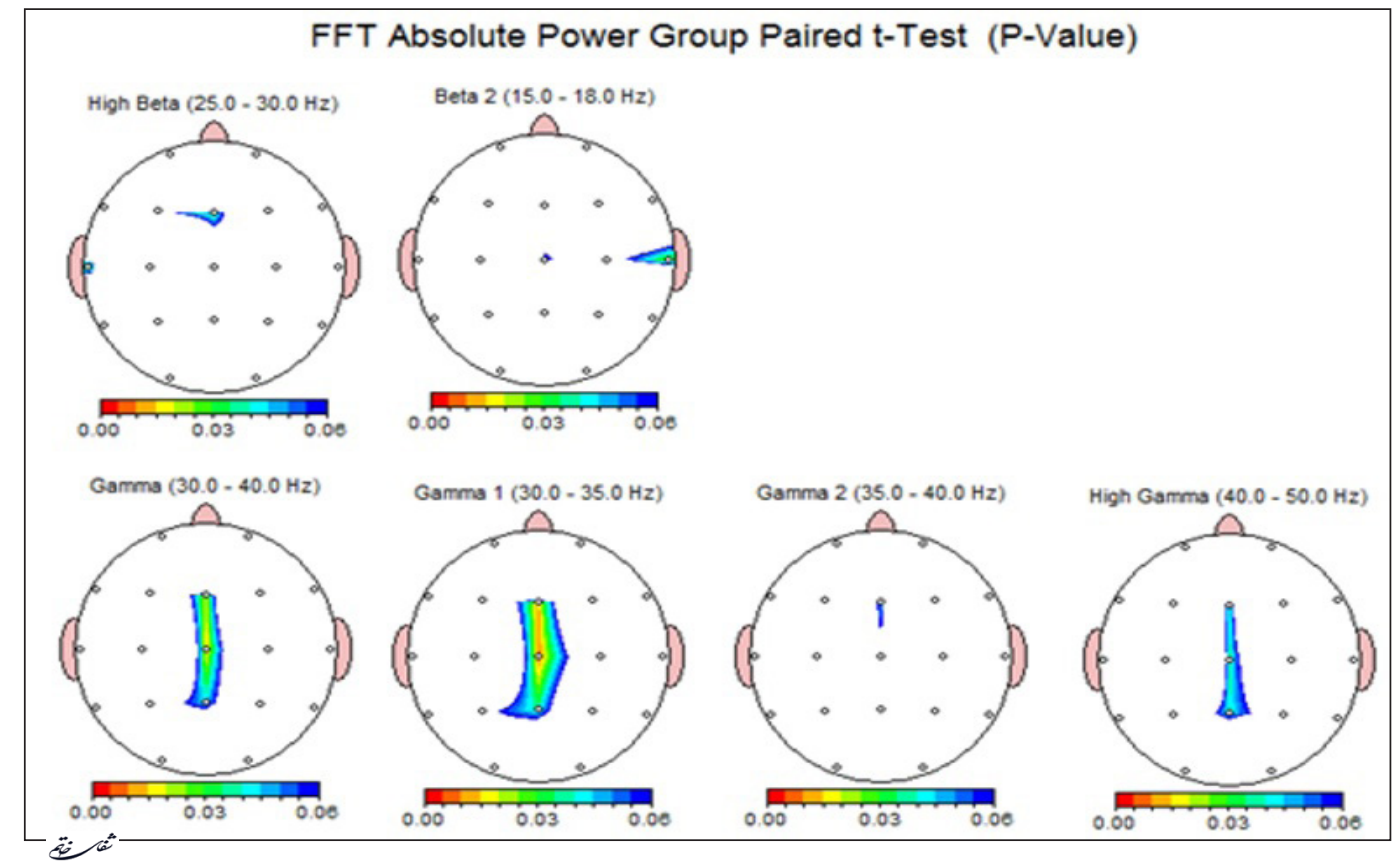

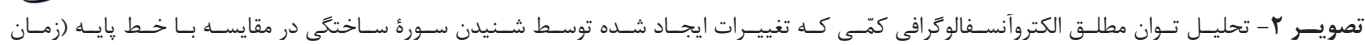

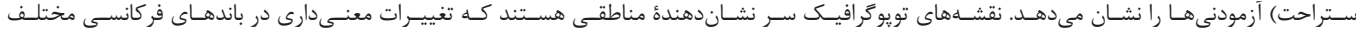

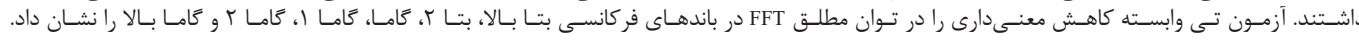
ايسن تغييـرات عمدتـاءدر نواحسى مركـزى روى داده اسـت.

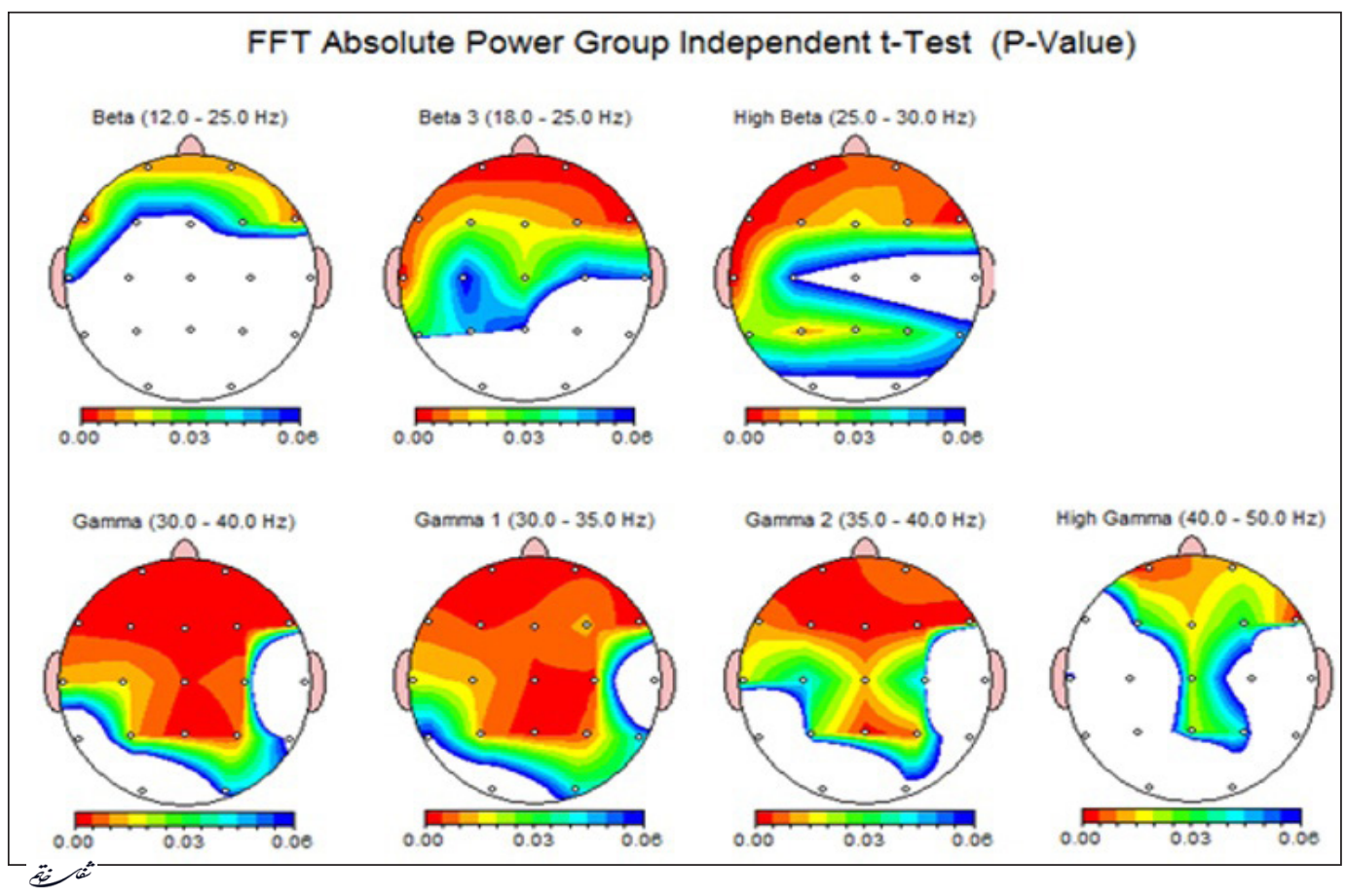

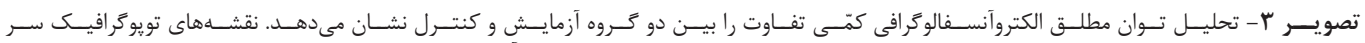

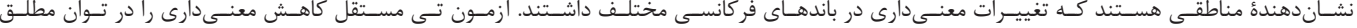

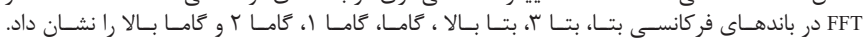

تعامـل وجــود دارد را مـورد تأييـد قـرار داد.

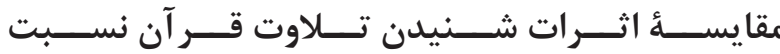

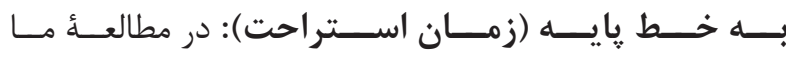

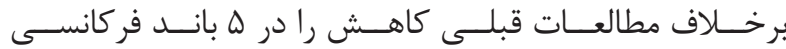

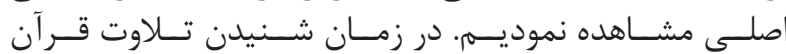

بحث و نتيجه

ايـن يزروهـش بــا هــدف تعييـن مبنــاى زيسـتى تأثير شـــيدن

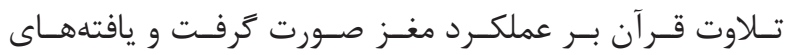

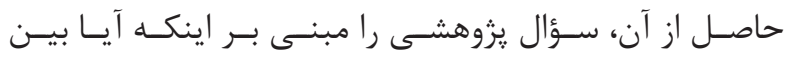

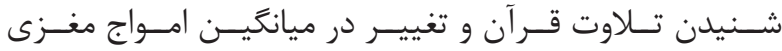




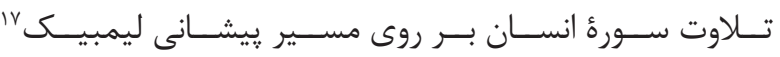

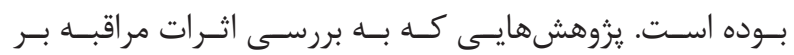

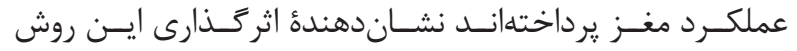

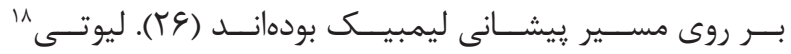

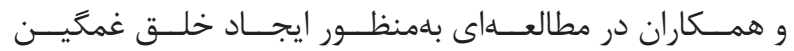

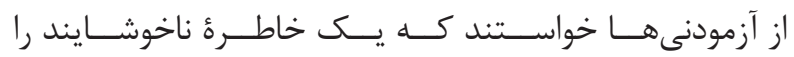

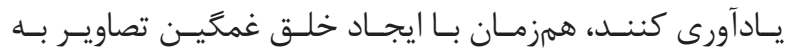

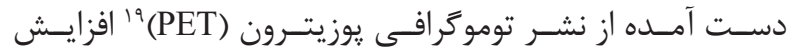

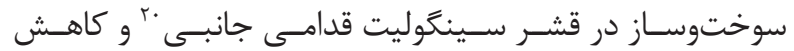

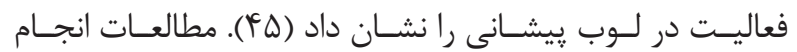

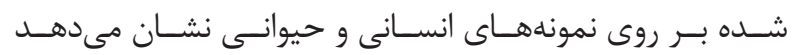

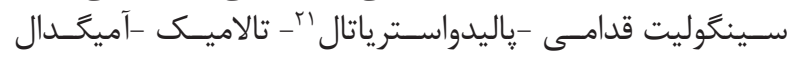

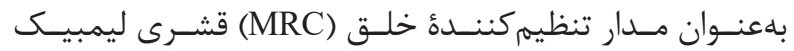

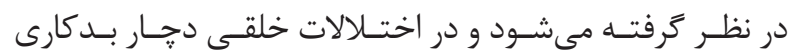

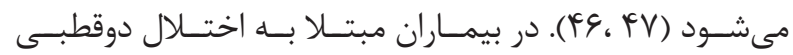

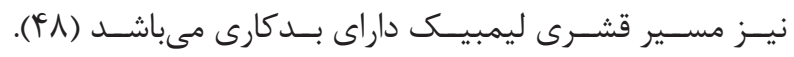

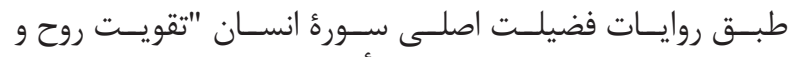

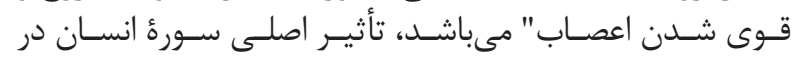

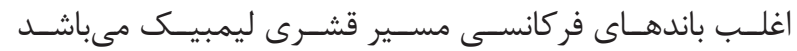

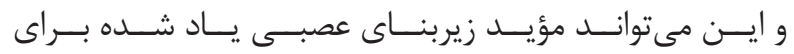

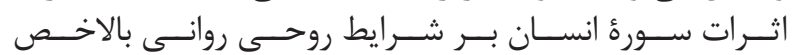

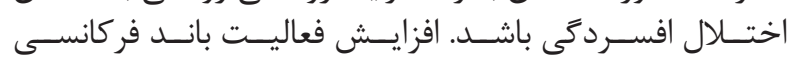

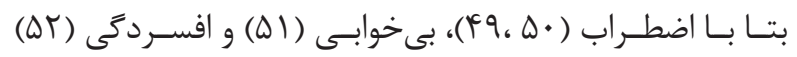

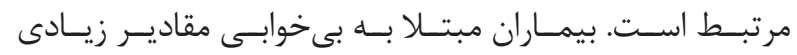

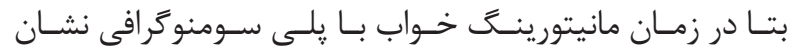

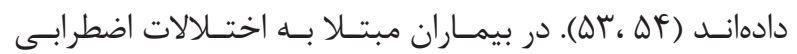

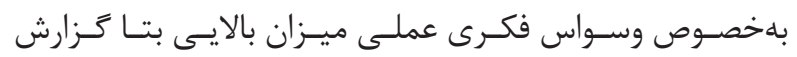

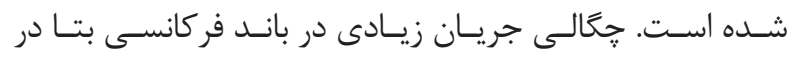

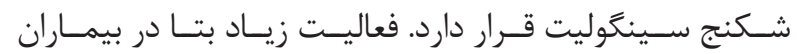

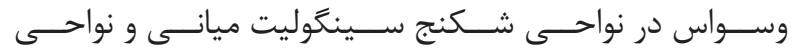

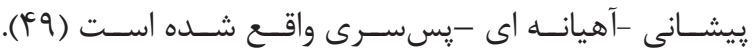

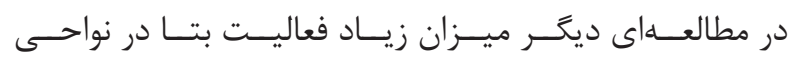

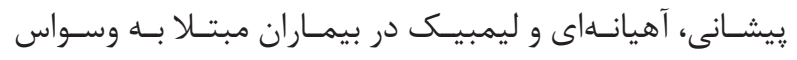

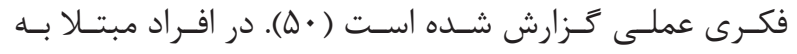

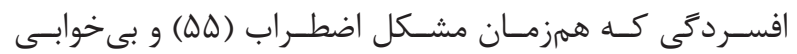

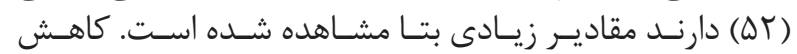

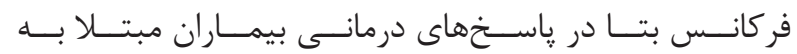

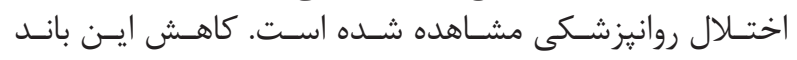

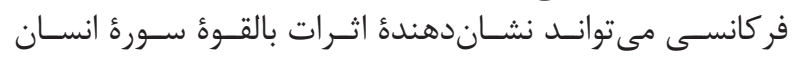

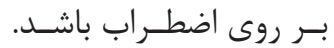

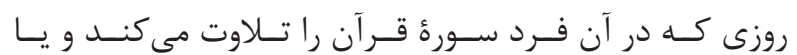

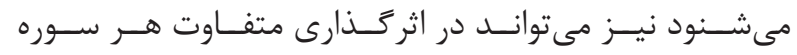

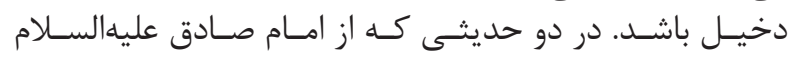

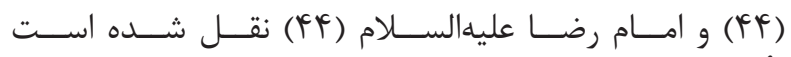

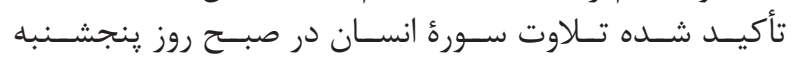

${ }^{17}$ Fronto-limbic

${ }^{18}$ Liotti

${ }^{19}$ Positron emission tomography

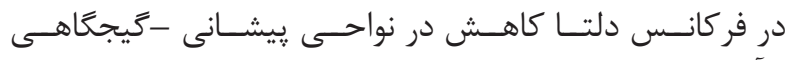

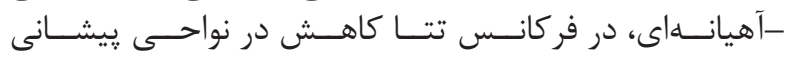

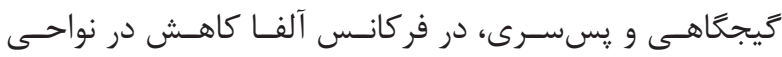

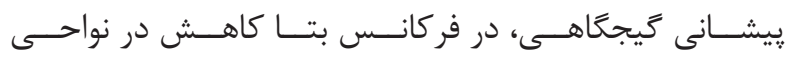

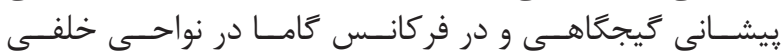

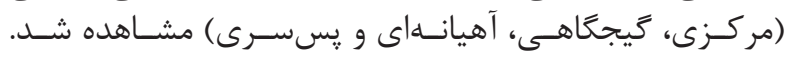

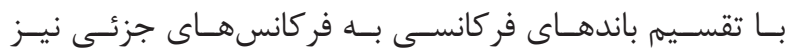

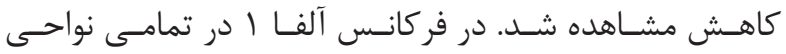

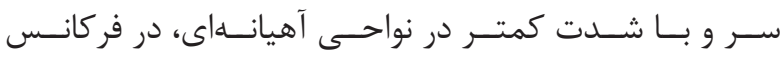

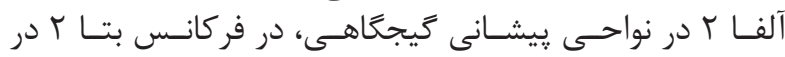

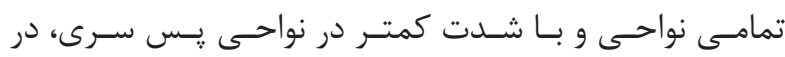

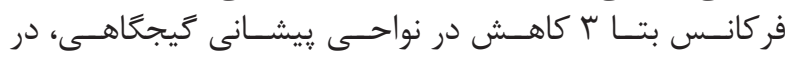

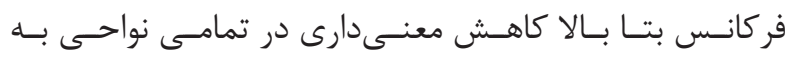

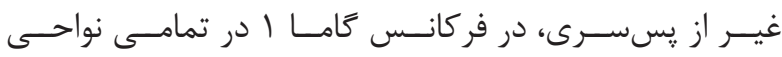

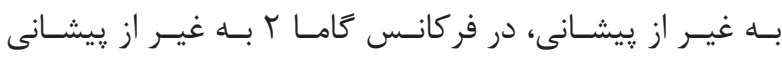

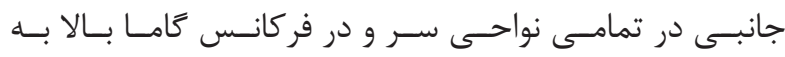

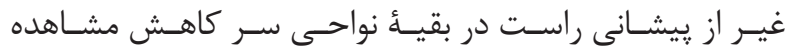

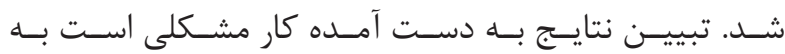

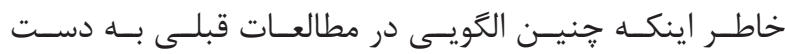

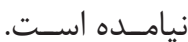

تعـداد محــدودى از مطالعـات به بررسـى اثـرات شـــيدن تلاوت

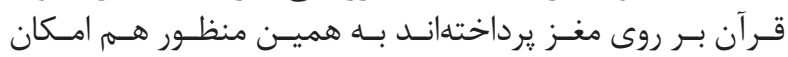

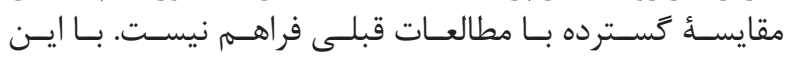

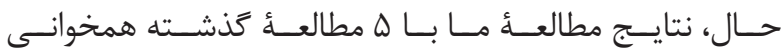

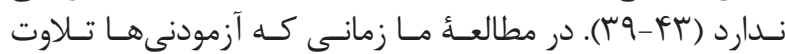

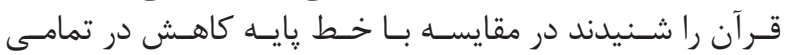

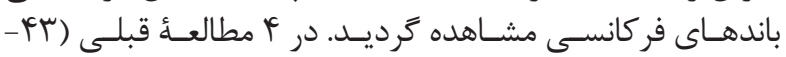

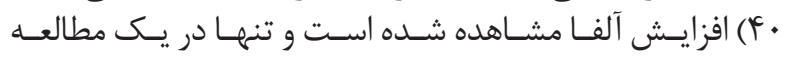

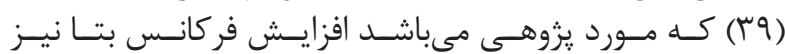

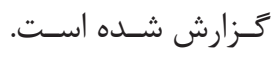

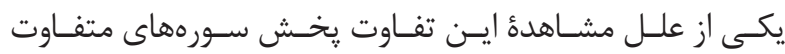

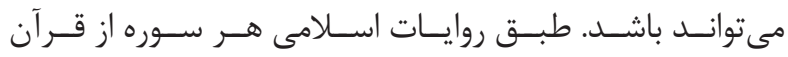

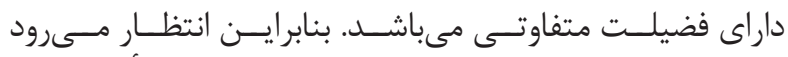

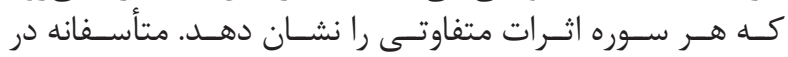

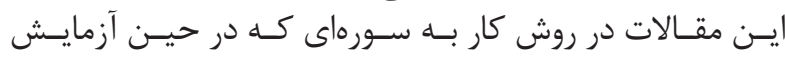

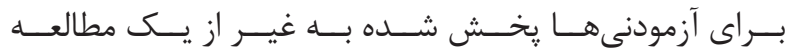

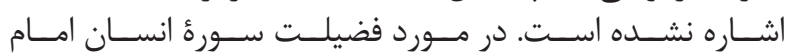

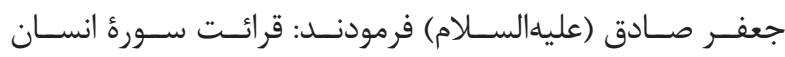

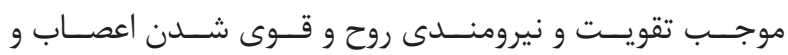

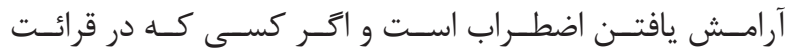

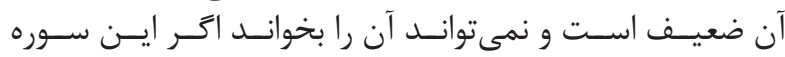

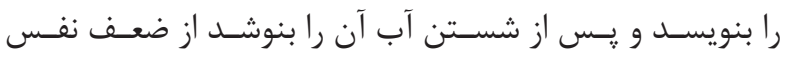

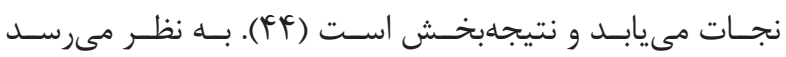

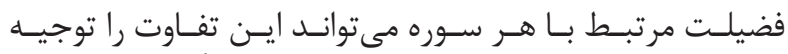

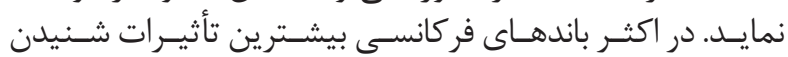

${ }^{20}$ Ventral anterior cingulate cortex

${ }^{21}$ Pallidostriatal 


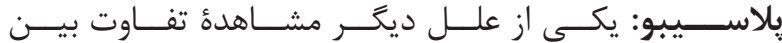

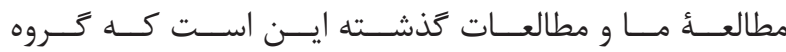

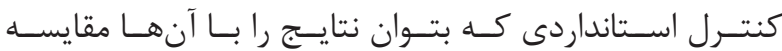

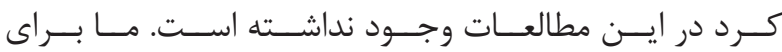

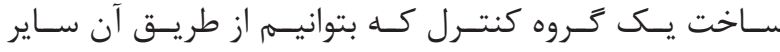

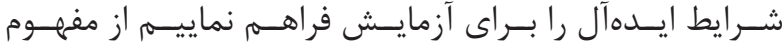

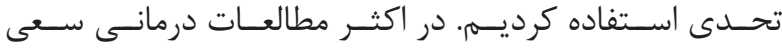

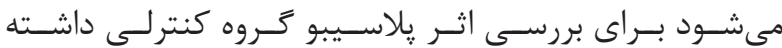

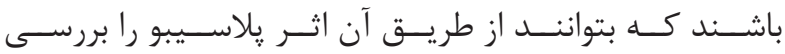

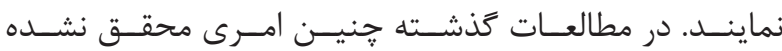

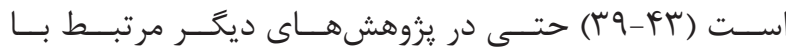

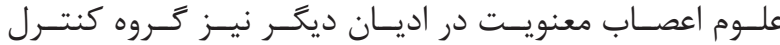

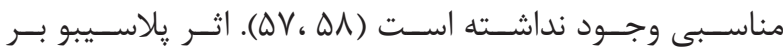

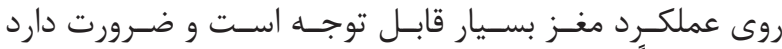

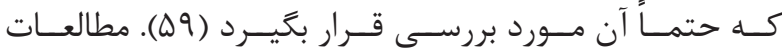

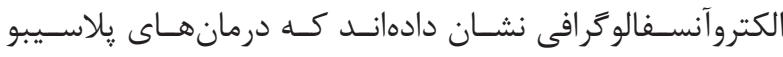

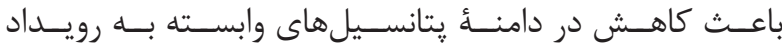

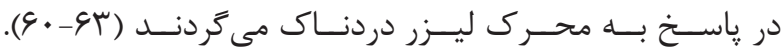

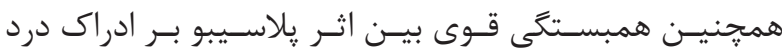

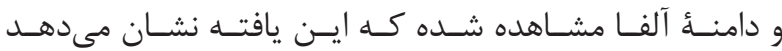

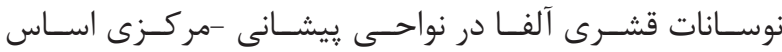

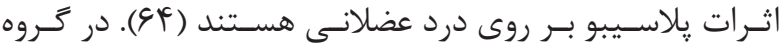

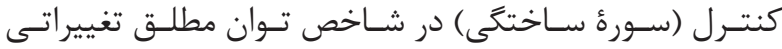

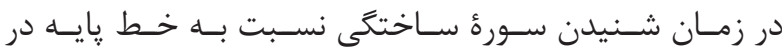

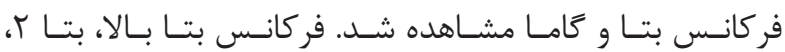

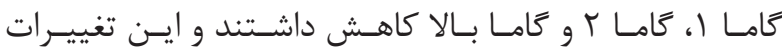

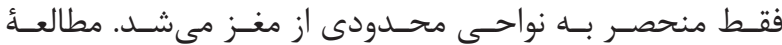

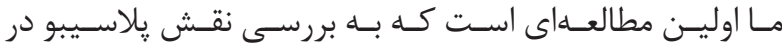

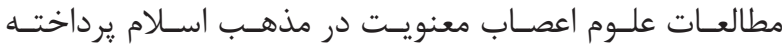

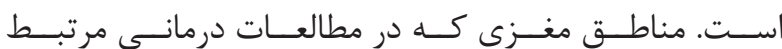

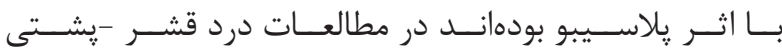

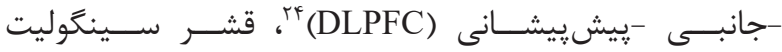

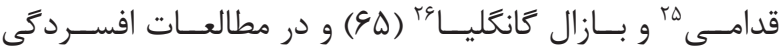

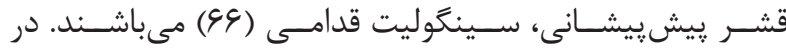

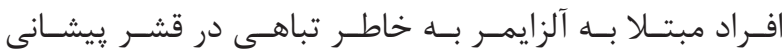

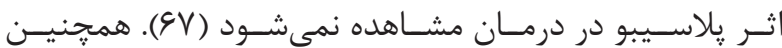

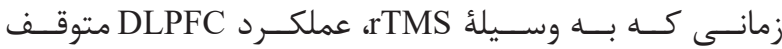

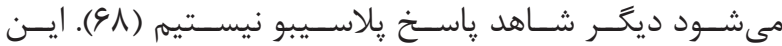

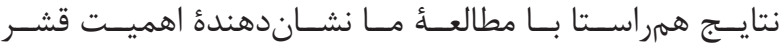

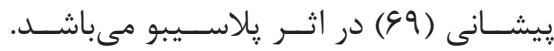

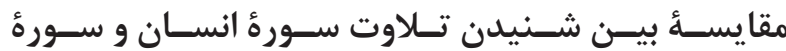

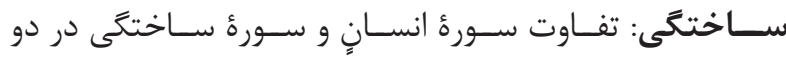

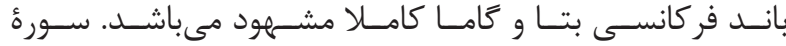

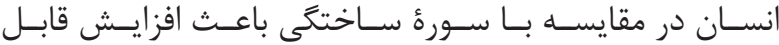

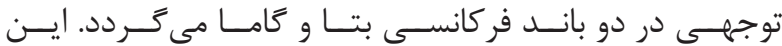

${ }^{22}$ Pascual-Leone

${ }^{23}$ Transcranial magnetic stimulation

${ }^{24}$ Dorsolateral prefrontal cortex

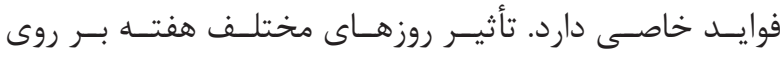

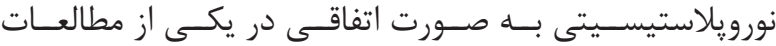

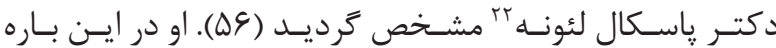

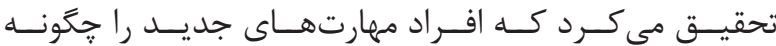

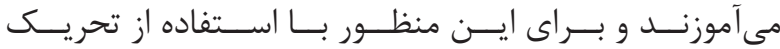

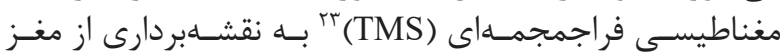

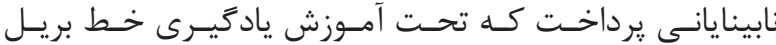

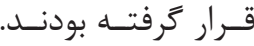

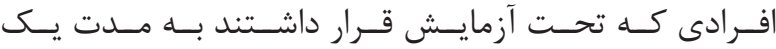

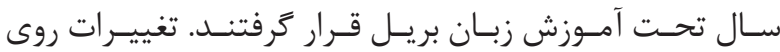

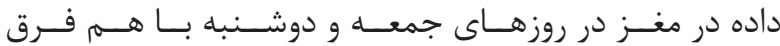

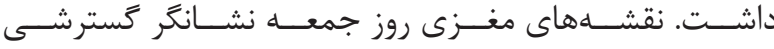

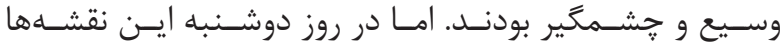

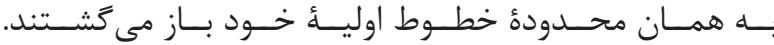

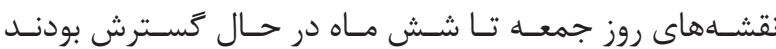

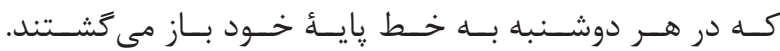

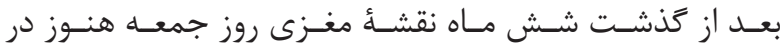

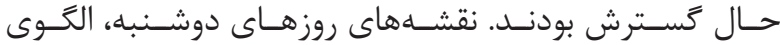

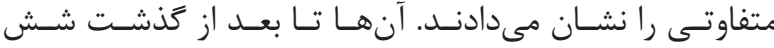

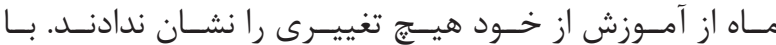

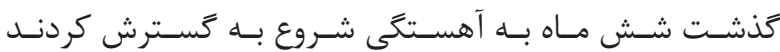

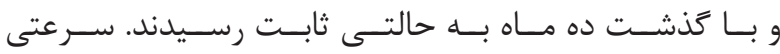

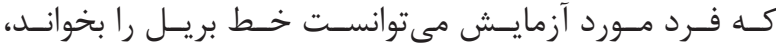

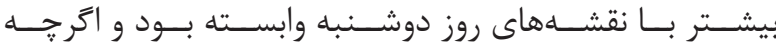

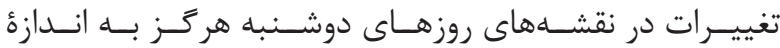

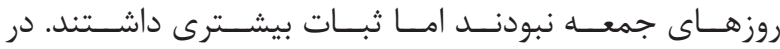

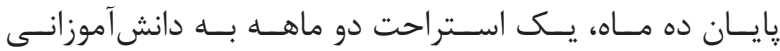

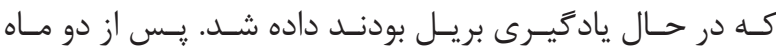

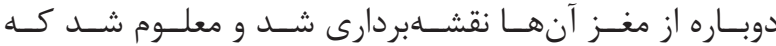

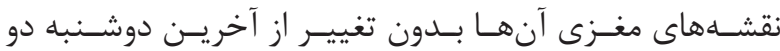

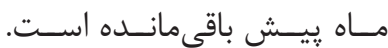

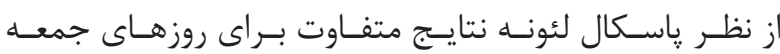

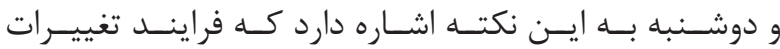

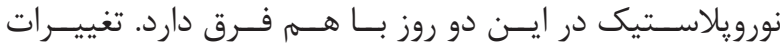

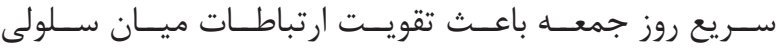

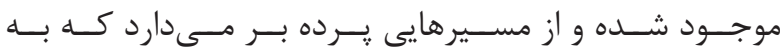

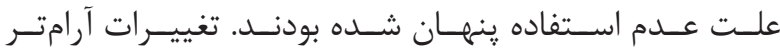

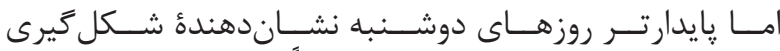

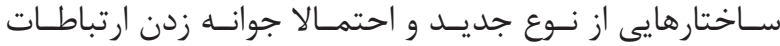

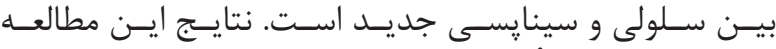

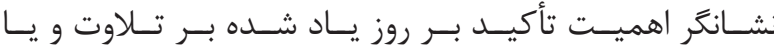

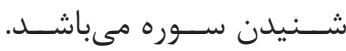

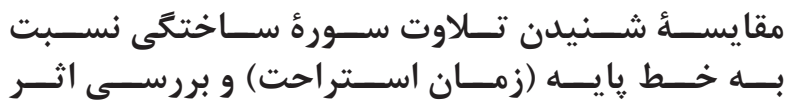
${ }^{25}$ Anterior cingulate

${ }^{26}$ Basal ganglia 


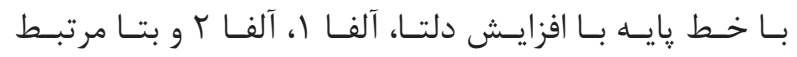

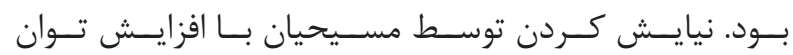

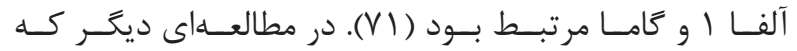

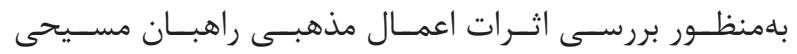

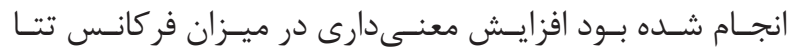

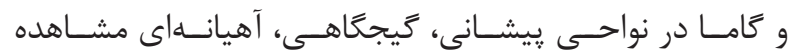

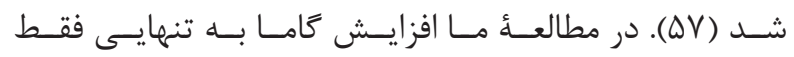

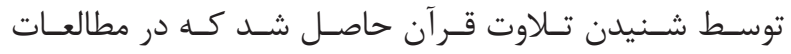

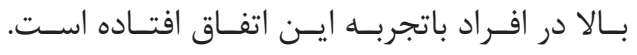

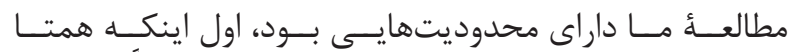

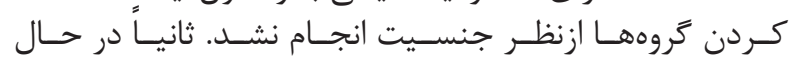

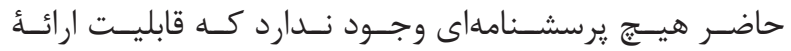

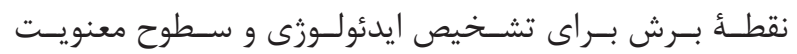

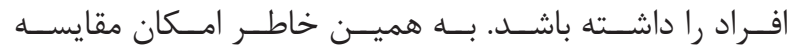

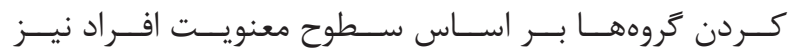

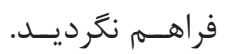

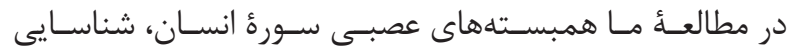

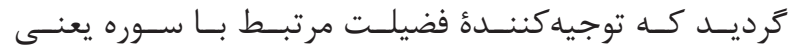

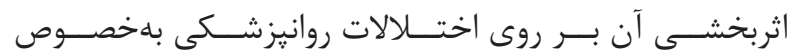

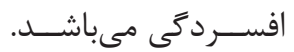

1. Davidian H, Izadi S, Nehaptian V, Motabar M. Preliminary study on the prevalence of mental disorders in the Caspian region. Iranian Journal of Public Health. 1974; 3(4): 145-56.

2. Noorbala AA, Bagheri Yazdi SA, Vaez mahdavi MR, Asadi lari M, Faghihzadeh S, Mohammad k, et al. Mental health changes in tehran during 12 years: comparing national health and disease survey (1999) and urban heart-2 project (2011). Daneshvar. 2014; 22(113): 37-44.

3. Noorbala AA, Mohammad k, Bagheri Yazdi SA, Yasami MT. Mental health status of individuals fifteen years and older in islamic repulic of Iran (2009). Health System Research Journal Hakim. 2002; 5(1): 1-10.

4. Kawakami N, Takeshima T, Ono Y, Uda H, Hata Y, Nakane Y, et al. Twelve-month prevalence, severity, and treatment of common mental disorders in communities in Japan: preliminary finding from the world mental health Japan survey 2002-2003. Psychiatry Clin Neurosci. 2005; 59(4): 441-52.

5. Kessler RC, McGonagle KA, Zhao S, Nelson CB, Hughes M, Eshleman S, et al. Lifetime and 12-month prevalence of DSM-III-R psychiatric disorders in the United States: results from the national comorbidity survey. Arch Gen Psychiatry. 1994; 51(1): 8-19.

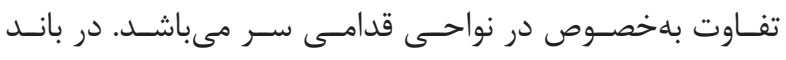

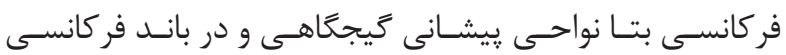

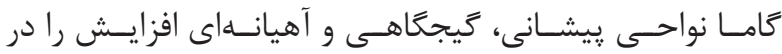

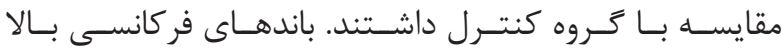

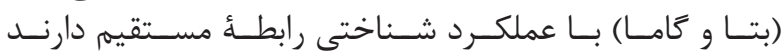

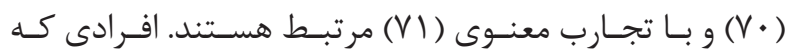

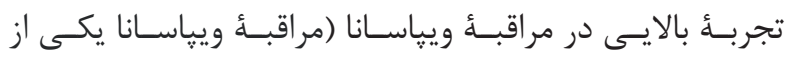

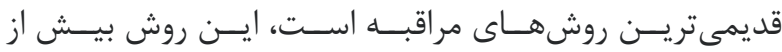

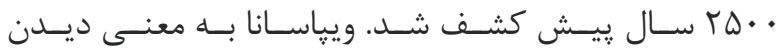

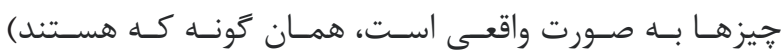

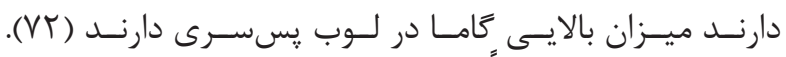

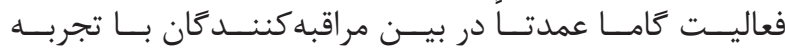

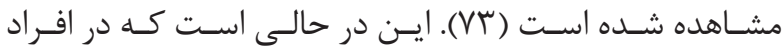

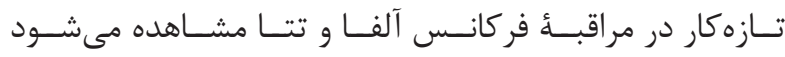

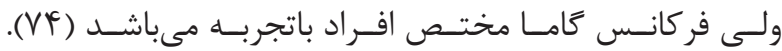

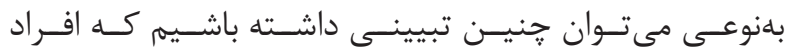

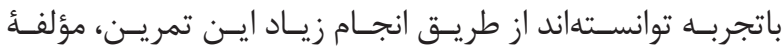

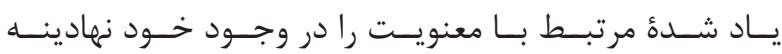

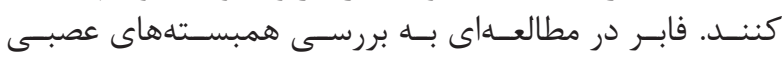

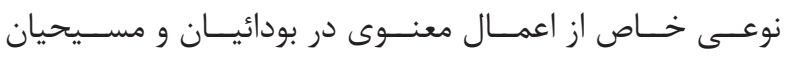

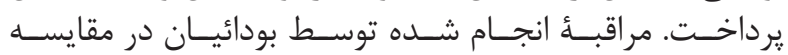

منابع

6. Fava M, Davidson KG. Definition and epidemiology of treatment-resistant depression. Psychiatr Clin North Am. 1996; 19(2): 179-200.

7. Mueller TI, Leon AC, Keller MB, Solomon DA, Endicott J, Coryell W, et al. Recurrence after recovery from major depressive disorder during 15 years of observational follow-up. Am J Psychiatry. 1999; 156(7): 1000-6.

8. Benkert O, Kepplinger H, Sobota K. Psychopharmaka im widerstreit. eine studie zur akzeptanz von psychopharmaka und zur darstellung in den medien. Berlin/Heidelberg: Springer; 1995.

9. Razali S. Complementary treatment of mental illness in Southeast Asia. International Medical Journal. 2000; 7(3): 189-91.

10. Ndetei D. Traditional healers and provision of mental health services in cosmopolitan informal settlements in Nairobi, Kenya. Afr J Psychiatry (Johannesbg). 2013; 16(2): 134-40.

11. Latypov A. Healers and psychiatrists: the transformation of mental health care in Tajikistan. Transcult Psychiatry. 2010; 47(3): 419-51.

12. Kurihara T, Kato M, Reverger R, Tirta IGR. Pathway 
to psychiatric care in Bali. Psychiatry Clin Neurosci. 2006; 60(2): 204-10.

13. Nortje G, Oladeji B, Gureje O, Seedat S. Effectiveness of traditional healers in treating mental disorders: a sys tematic review. Lancet Psychiatry. 2016; 3(2): 154-70.

14. Plante TG, Sherman AC. Faith and health: psychological perspectives: Guilford Press; 2001.

15. Levin JS. How religion influences morbidity and health: reflections on natural history, salutogenesis and host resistance. Soc Sci Med. 1996; 43(5): 849-64.

16. Koenig HG, McCullough ME, Larson DB. Handbook of religion and health: Oxford University Press; 2001.

17. Larson DB, Milano M. Making the case for spiritual interventions in clinical practice. Mind Body Med. 1997; 2: 20-30.

18. Koenig HG. Handbook of religion and mental health: Elsevier; 1998.

19. George LK, Larson DB, Koenig HG, McCullough ME. Spirituality and health: what we know, what we need to know. J Soc Clin Psychol. 2000; 19(1): 102-16.

20. Miller WR. Researching the spiritual dimensions of alcohol and other drug problems. Addiction. 1998; 93(7): 979-90.

21. Kendler KS, Gardner CO, Prescott CA. Religion, psychopathology, and substance use and abuse: a multimeasure, genetic-epidemiologic study. Am J Psychiatry. 1997; 154(3): 322-9.

22. Gorsuch RL. Religious aspects of substance abuse and recovery. J Soc Issues. 1995; 51(2): 65-83.

23. Gartner J, Larson DB, Allen GD. Religious commitment and mental health: a review of the empirical literature. J Psychol Theol. 1991; 19: 6-25.

24. Aaen-Stockdale C. Neuroscience for the Soul. Psychologist. 2012; 25(7): 520-3.

25. Beauregard M, Paquette V. Neural correlates of a mystical experience in carmelite nuns. Neurosci Lett. 2006; 405(3): 186-90.

26. Hölzel BK, Lazar SW, Gard T, Schuman-Olivier Z, Vago DR, Ott U. How does mindfulness meditation work? proposing mechanisms of action from a conceptual and neural perspective. Perspect Psychol Sci. 2011; 6(6): 537-59.
27. Cahn BR, Polich J. Meditation states and traits: EEG, ERP, and neuroimaging studies. Psychol Bull. 2006; 132(2): 180 .

28. Kasamatsu A, Hirai T. An electroencephalographic study on the zen meditation (Zazen). Folia Psychiatr Neurol Jpn. 1966; 20(4): 315-36.

29. Lagopoulos J, Xu J, Rasmussen I, Vik A, Malhi GS, Eliassen $\mathrm{CF}$, et al. Increased theta and alpha EEG activity during nondirective meditation. J Altern Complement Med. 2009; 15(11): 1187-92.

30. Wong W, Camfield DA, Woods W, Sarris J, Pipingas A. Spectral power and functional connectivity changes during mindfulness meditation with eyes open: a magnetoencephalography (MEG) study in long-term meditators. Int J Psychophysiol. 2015; 98(1): 95-111.

31. Lomas T, Ivtzan I, Fu CH. A systematic review of the neurophysiology of mindfulness on EEG oscillations. Neurosci Biobehav Rev. 2015; 57: 401-10.

32. Shirvani M, Mirzaeian R, Ghaderi A. The effect of holy quran sound on vital sign and spo2 in unconscious patients hospitalized in the ICU. $3^{\text {th }}$ Provincial Congress on Quran and Health; Iran. 18-23: Shahrekord University of Medical Sciences; 2013. p. 18-21.

33. Soleimani M. The study of recitation of the Holy Quran on levels of counciousness patients with coma caused by a cerebral injury: Tarbiat Modares University; 1998 .

34. Keshavars M, Eskandaril N, Jahdi F, Ashaieri H, Hoseini F, Kalani M. The effect of holly Quran recitation on physiological responses of premature infant. koomesh Journal. 2010; 11(3): 169-77.

35. Forouhari S, Honarvaran R, Maasoumi R, Robati M, Hashem zadeh I, Setayesh Y. Evaluation of the auditory effects of the sound of Quarn e Karim on labor pain. Quran and Medicine. 2011; 1(2): 14-8.

36. Sahmeddini MA, Zamani Lari M, Nabi Rahimian M, Danaei L, Beigi N, Habibi H. The effect of listening to the Quran on serum cortisol levels and anxiety in primiparous women during the first stage of labor. The Iranian Journal of Obsterics, Gynecology and Infertility. 2014; 17(99): 1-7.

37. Ghasem Tabar N, Fayyaz I, Ghasem Tabar A, Omidi H. The effect of Quran recitation on the anxiety-before-exam of the students. Education Islamic. 2013; 8(16): 101-16.

38. Aghajani M, Mirbagher N. Comparing the effect of Holy Quran recitation and music on patient anxiety and 
vital signs before abdominal surgeries. Islamic Lifes tyle Centered on Health. 2012; 1(1): 66-84.

39. Alshaikhli IFT, Yahya SA, Pammusu I, Alarabi KF. A study on the effects of EEG and ECG signals while listening to Qur'an recitation. information and communication technology for the muslim world (ICT4M). The $5^{\text {th }}$ International Conference. 2014.

40. Shekha MS, Hassan AO, Othman SA. Effects of quran listening and music on electroencephalogram brain waves. Egypt J Exp Biol. 2013; 9(1): 1-7.

41. Zulkurnaini NA, Kadir RSSA, Murat ZH, Isa RM. The comparison between listening to al-Quran and lis tening to classical music on the brainwave signal for the alpha band. Intelligent systems, modelling and simulation (ISMS). $3^{\text {rd }}$ International Conference. 2012.

42. Kamal NF, Mahmood NH, Zakaria NA. Modeling brain activities during reading working memory task: comparison between reciting Quran and reading book. Procedia Soc Behav Sci. 2013; 97: 83-9.

43. Abdullah AA, Omar Z. The effect of temporal EEG signals while listening to Quran recitation. IJASEIT. 2011; 1(4): 372-5.

44. The virtues and properties of surah of Ensan. 2017. http://quran.anhar.ir/fazilat-307.htm.

45. Liotti M, Mayberg HS, Brannan SK, McGinnis S, Jerabek P, Fox PT. Differential limbic-cortical correlates of sadness and anxiety in healthy subjects: implications for affective disorders. Bio Psychiatry. 2000; 48(1): 42-30.

46. Mayberg HS. Modulating dysfunctional limbiccortical circuits in depression: towards development of brain-based algorithms for diagnosis and optimised treatment. Br Med Bull. 2003; 65(1): 193-207.

47. Drevets M, Wayne C. Functional neuroimaging s tudies of depression: the anatomy of melancholia. Annu Rev Med. 1998; 49(1): 341-61.

48. Anand A, Li Y, Wang Y, Lowe MJ, Dzemidzic M. Resting state corticolimbic connectivity abnormalities in unmedicated bipolar disorder and unipolar depression. Psychiatry Res: Neuroimaging. 2009; 171(3): 189-98.

49. Sherlin L, Congedo M. Obsessive-compulsive dimension localized using low-resolution brain electromagnetic tomography (LORETA). Neurosci Lett. 2005; 387(2): 72-4.
50. Velikova S, Locatelli M, Insacco C, Smeraldi E, Comi G, Leocani L. Dysfunctional brain circuitry in obsessive-compulsive disorder: source and coherence analysis of EEG rhythms. NeuroImage. 2010; 49(1): 977-83.

51. Perlis ML, Merica H, Smith MT, Giles DE. Beta EEG activity and insomnia. Sleep Med Rev. 2001; 5(5): 365-76.

52. Nofzinger EA, Price JC, Meltzer CC, Buysse DJ, Villemagne VL, Miewald JM, et al. Towards a neurobiology of dysfunctional arousal in depression: the relationship between beta EEG power and regional cerebral glucose metabolism during NREM sleep. Psychiatry Res. 2000; 98(2): 71-91.

53. Lamarche $\mathrm{CH}$, Ogilvie RD. Electrophysiological changes during the sleep onset period of psychophysiological insomniacs. psychiatric insomniacs, and normal sleepers. Sleep. 1997; 20(9): 726-33.

54. Perlis ML, Smith MT, Andrews PJ, Orff H, Giles DE. Beta/Gamma EEG activity in patients with primary and secondary insomnia and good sleeper controls. Sleep. 2001; 24(1): 110-7.

55. Pizzagalli DA, Nitschke JB, Oakes TR, Hendrick AM, Horras KA, Larson CL, et al. Brain electrical tomography in depression: the importance of symptom severity, anxiety, and melancholic features. Biological Psychiatry. 2002; 52(2): 73-85.

56. Doidge N. The brain that changes itself: stories of personal triumph from the frontiers of brain science: Penguin; 2007.

57. Beauregard M, Paquette V. EEG activity in carmelite nuns during a mystical experience. Neurosci Lett. 2008; 444(1): 1-4.

58. Beauregard M, Courtemanche J, Paquette V. Brain activity in near-death experiencers during a meditative $\mathrm{s}$ tate. Resuscitation. 2009; 80(9): 1006-10.

59. Wager TD, Atlas LY. The neuroscience of placebo effects: connecting context, learning and health. Nat Rev Neurosci. 2015; 16(7): 403-18.

60. Watson A, El-Deredy W, Vogt BA, Jones AK. Placebo analgesia is not due to compliance or habituation: EEG and behavioural evidence. Neuroreport. 2007; 18(8): 771-5.

61. Wager TD, Matre D, Casey KL. Placebo effects in laser-evoked pain potentials. Brain Behav Immun. 
2006; 20(3): 219-30.

62. Nakamura Y, Donaldson GW, Kuhn R, Bradshaw $\mathrm{DH}$, Jacobson RC, Chapman CR. Investigating dose-dependent effects of placebo analgesia: a psychophysiological approach. Pain. 2012; 153(1): 227 37.

63. Lorenz J, Hauck M, Paur RC, Nakamura Y, Zimmermann R, Bromm B, et al. Cortical correlates of false expectations during pain intensity judgments-a possible manifestation of placebo/nocebo cognitions. Brain Behav Immun. 2005; 19(4): 283-95.

64. Li L, Wang H, Ke X, Liu X, Yuan Y, Zhang D, et al. Placebo analgesia changes alpha oscillations induced by tonic muscle pain: EEG frequency analysis including data during pain evaluation. Front Comput Neurosci. 2016; 10: 45. doi: 10.3389/fncom.2016.00045.

65. Oken BS. Placebo effects: clinical aspects and neurobiology. Brain. 2008; 131(11): 2812-23.

66. Mayberg HS, Silva JA, Brannan SK, Tekell JL, Mahurin RK, McGinnis S, et al. The functional neuroanatomy of the placebo effect. Am J Psychiatry. 2002; 159(5): 728-37.

67. Benedetti F, Arduino C, Costa S, Vighetti S, Tarenzi L, Rainero I, et al. Loss of expectation-related mechanisms in Alzheimer's disease makes analgesic therapies less effective. Pain. 2006; 121(1): 133-44.
68. Krummenacher P, Candia V, Folkers G, Schedlowski M, Schönbächler G. Prefrontal cortex modulates placebo analgesia. Pain. 2010; 148(3): 368-74.

69. Parris BA. The prefrontal cortex and suggestion: hypnosis vs. placebo effects. Front Psychol. 2016; 7: 415. doi: 10.3389/fpsyg.2016.00415.

70. Bosman CA, Lansink CS, Pennartz CM. Functions of gamma-band synchronization in cognition: from single circuits to functional diversity across cortical and subcortical systems. Eur J Neurosci. 2014; 39(11): 1982-99.

71. Faber EL. The neural correlates of two forms of spiritual love: an EEG study. BioRxiv. 2016. doi: https:// doi.org/10.1101/045898.

72. Cahn BR, Delorme A, Polich J. Occipital gamma activation during vipassana meditation. Cognitive Processing. 2010; 11(1): 39-56.

73. Fell J, Axmacher N, Haupt S. From alpha to gamma: electrophysiological correlates of meditation-related $\mathrm{s}$ tates of consciousness. Med Hypotheses. 2010; 75(2): 218-24.

74. Lutz A, Greischar LL, Rawlings NB, Ricard M, Davidson RJ. Long-term meditators self-induce highamplitude gamma synchrony during mental practice. Proc Natl Acad Sci U S A. 2004; 101(46): 16369-73. 